\title{
Experimentally accessible orbits near a Bykov cycle
}

\author{
Roberto Barrio \\ Departamento de Matemática Aplicada and IUMA, Universidad de Zaragoza, E-50009 Zaragoza, Spain \\ rbarrio@unizar.es \\ Maria Carvalho \\ Centro de Matemática da Univ. do Porto, Rua do Campo Alegre, 687, 4169-007 Porto, Portugal \\ mpcarval@fc.up.pt \\ M. Luísa Castro \\ INESC TEC Porto and Center for Health Technology and Services Research (CINTESIS), Faculdade de \\ Medicina da Universidade do Porto, Portugal \\ luisacastro@med.up.pt \\ Alexandre A. P. Rodrigues \\ Centro de Matemática da Univ. do Porto, Rua do Campo Alegre, 687, 4169-007 Porto, Portugal \\ alexandre.rodrigues@fc.up.pt
}

Received (to be inserted by publisher)

\begin{abstract}
This paper reports numerical experiments done on a two-parameter family of vector fields which unfolds an attracting heteroclinic cycle linking two saddle-foci. We investigated both local and global bifurcations due to symmetry breaking in order to detect either hyperbolic or chaotic dynamics. Although a complete understanding of the corresponding bifurcation diagram and the mechanisms underlying the dynamical changes is still out of reach, using a combination of theoretical tools and computer simulations we have uncovered some complex patterns. We have selected suitable initial conditions to analyze the bifurcation diagrams, and regarding these solutions we have located: (A) an open domain of parameters with regular dynamics; (B) infinitely many parabolic-type curves associated to homoclinic Shilnikov cycles which act as organizing centers; (C) a crisis region related to the destruction or creation of chaotic attractors; (D) a large Lebesgue measure set of parameters where chaotic regimes are dominant, though sinks and chaotic attractors may coexist, and in whose complement we observe shrimps.
\end{abstract}

Keywords: Bifurcation; Bykov attractor; Homoclinic and heteroclinic cycle; multistability; biparametric diagrams.

\section{Introduction}

Several problems in the study of dynamical chaos are prompted by the following question: What are the mechanisms of transition from regular to chaotic dynamics? Global bifurcations of invariant sets, such as competition between attractors or metamorphoses within basin boundaries, are the most easily detected dynamical phenomena and probably the most often described in the literature. As suggested in [Gonchenko et al., 1996], the transition to chaos is most likely connected with intricate mathematical invariant sets, nowadays known as quasi-stochastic attractors, such as limiting sets containing nontrivial hyperbolic com- 
ponents coexisting with stable periodic solutions from which the former cannot be disconnected by an isotopy.

The inherent stochastic behavior in this setting is hard to unravel since there are no theoretical tools to predict the location and structure of chaotic regions of the phase space. Yet the complexity of a system may be glimpsed through either its entropy or its Lyapunov exponents. A Lyapunov exponent associated to a solution of a differential equation is an average exponential rate of divergence or convergence of nearby orbits in the phase space. As they measure the rate at which the dynamics creates or destroys information, the Lyapunov exponents, equal in number to the dimension of the phase space, should allow one to distinguish between chaos and stable periodicity. A positive exponent reflects the existence of a direction in which the system experiences the repeated stretching and folding that mixes nearby states on the attractor. Thus, the long-term behavior of an initial condition with a positive Lyapunov exponent cannot be predicted, and this is one of the most common traits of chaos. Unsurprisingly, this is also the key idea behind several numerical experiments with chaotic dynamical systems [Barrio et al., 2011]. Indeed, since nearby solutions may correspond to numerical almost identical states, the presence of exponential orbital divergence implies that trajectories whose initial conditions are hard to distinguish will soon depart, and most likely behave afterwards quite differently. Although our predictive ability may be rapidly lost and the presence of non-zero Lyapunov exponents does not quantify in general a robust dynamical behavior, the study of the number of positive Lyapunov exponents along well chosen orbits motivates one of the most powerful computational techniques available to build a reliable approximation of a bifurcation diagram. We refer the reader to [Wolf et al., 1985] for more information on what dynamical knowledge is conveyed from the magnitudes of the Lyapunov exponents for a family of vector fields in dimension three or four.
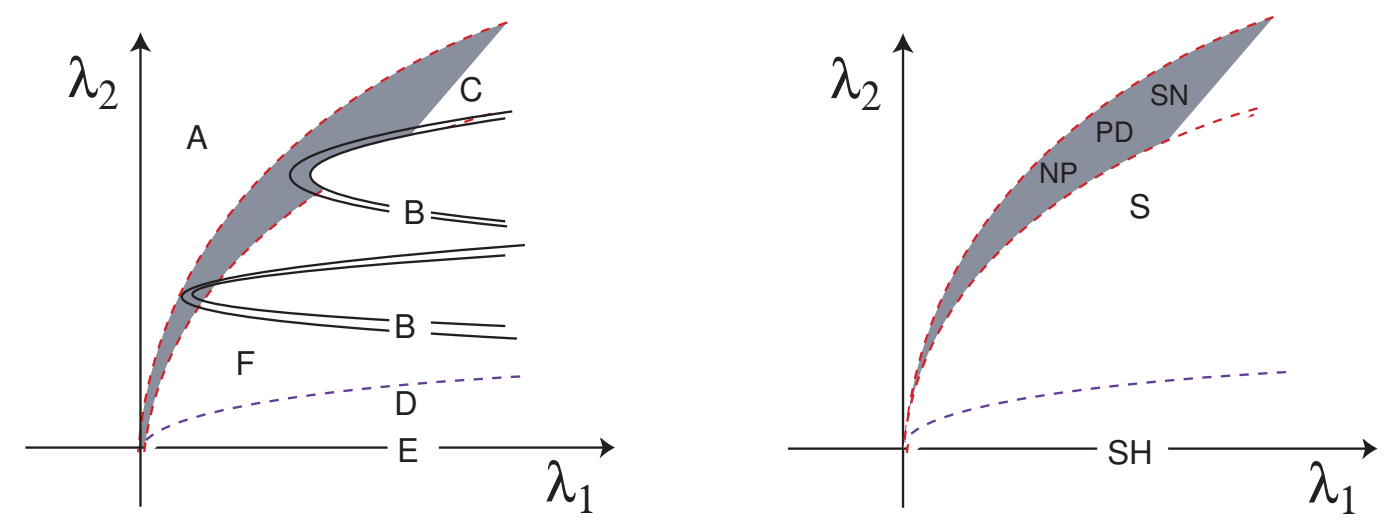

Fig. 1. Detected features on the dynamics (left) and bifurcations (right). (A) Periodic solution. (B) Homoclinic cycles of Shilnikov type within cocoon structures, and multi-pulses. (C) Crisis region, showing the appearance and destruction of chaos. (D) Hyperbolic dynamics, such as horseshoes and sinks, besides strange attractors. (E) Saturated horseshoes (SH), Newhouse phenomena (NP), saddle-node (SN) and period doubling (PD) bifurcations. (S) Shrimps.

Directions along which the flow is, on the average, expanding (respectively contracting) correspond to positive (respectively negative) exponents. Moreover, the sum of the Lyapunov exponents is the timeaveraged divergence of the phase space vector field. Hence a dissipative flow has at least one negative Lyapunov exponent, the sum of all of its exponents is negative and, for Lebesgue almost all initial conditions, the post-transient motion of the trajectories will occur in a zero volume limit set.

We have performed several computer experiments using the packages of Dstool [Guckenheimer et $a l .$, 1997] and Matlab - MATLAB (R2017b, MathWorks, Natick, MA, USA) for a two-parameter family of vector fields introduced in [Swift, 1988)] and explored in [Aguiar et al., 2006]. The periodic or chaotic nature of solutions could only be determined on a case-by-case examination by fixing parameters and investigating the dynamics for well chosen initial conditions. Additional care was needed while interpreting the numerical integration of these flows since, for some parameters, they exhibit quasi-stochastic attractors and these are prone to rounding errors that may ruin the simulations. The content of the paper is summarized in Figure 1, 

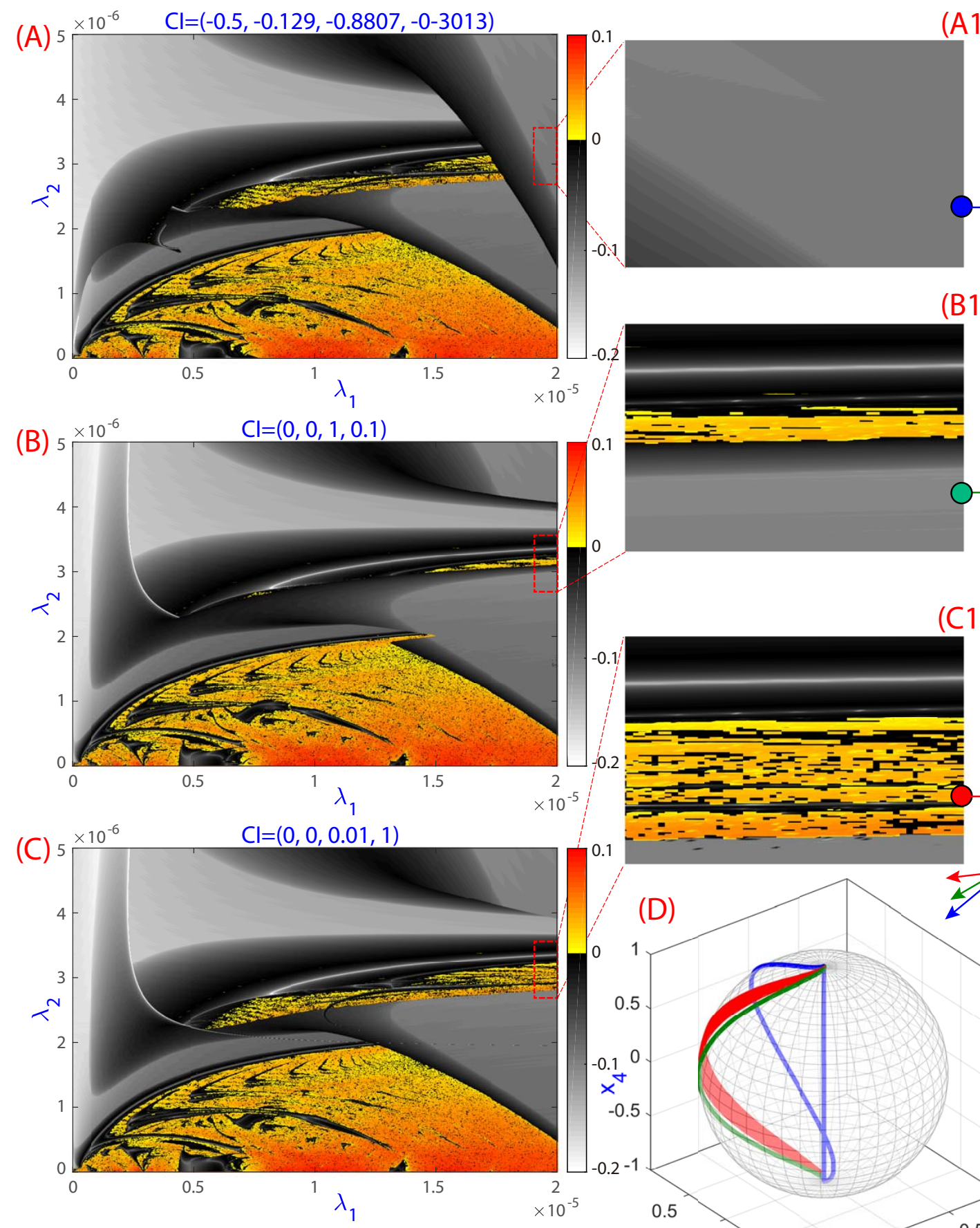

0.1

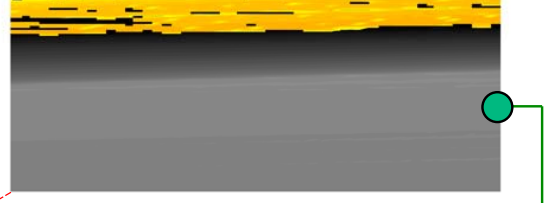

(C1)

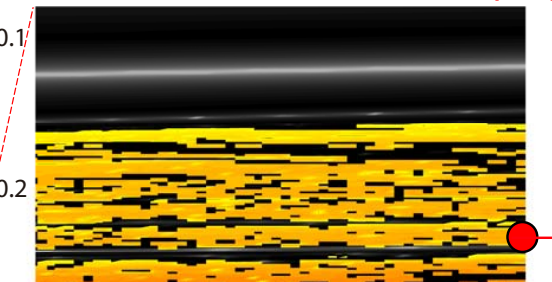

0.1

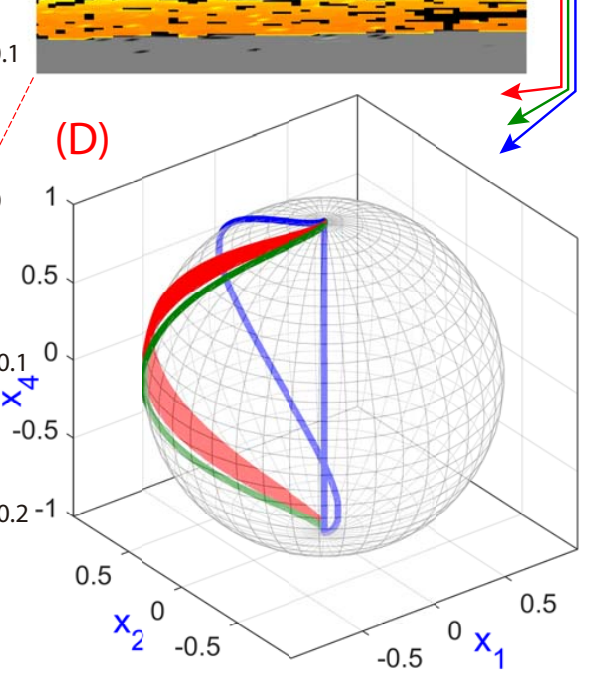

Fig. 2. Lyapunov exponents biparametric diagram in the domain $\left(\lambda_{1}, \lambda_{2}\right) \in\left[0,2 \times 10^{-5}\right] \times\left[0,5 \times 10^{-6}\right]$ for the initial conditions CI4: $=(0.5,-0.129,-0.8807,0.3013)$, CI $5:=(0,0,1,0.1)$ and CI $:=(0,0,0.01,1) \approx \mathrm{CI} 1$, and fixed parameters $\alpha_{1}=2.5$ and $\alpha_{2}=-0.1$. Each point is colored according to the sign of the first two Lyapunov exponents of the orbit (if the first exponent is positive it is painted in yellow-red colors; if it is zero, the second exponent is painted in grey scale). The plots exhibit multistability behaviour as shown more clearly in the magnifications where different structures are observed. For instance, choosing the parameters $\lambda_{1}=2 \times 10^{-5}$ and $\lambda_{2}=3 \times 10^{-6}$ we observe the coexistence of two periodic orbits attracting the two initial conditions CI4 and CI5 (blue and green dots), which are close to the one-dimensional connection; and one chaotic attractor (red dots) using the initial condition CI1.

sketched after several simulations as the ones of Figure 2 . 


\section{The model}

For $\lambda_{1}, \lambda_{2} \in[0,1]$, our object of study is the two-parameter family of vector fields on $\mathbb{R}^{4}$

$$
x=\left(x_{1}, x_{2}, x_{3}, x_{4}\right) \in \mathbb{S}^{3} \mapsto f_{\left(\lambda_{1}, \lambda_{2}\right)}(x)
$$

defined for each $x=\left(x_{1}, x_{2}, x_{3}, x_{4}\right)$ by

$$
\left\{\begin{array}{l}
\dot{x}_{1}=x_{1}\left(1-r^{2}\right)-x_{2}-\alpha_{1} x_{1} x_{4}+\alpha_{2} x_{1} x_{4}^{2}+\lambda_{2} x_{3}^{2} x_{4} \\
\dot{x}_{2}=x_{2}\left(1-r^{2}\right)+x_{1}-\alpha_{1} x_{2} x_{4}+\alpha_{2} x_{2} x_{4}^{2} \\
\dot{x}_{3}=x_{3}\left(1-r^{2}\right)+\alpha_{1} x_{3} x_{4}+\alpha_{2} x_{3} x_{4}^{2}+\lambda_{1} x_{1} x_{2} x_{4}-\lambda_{2} x_{1} x_{3} x_{4} \\
\dot{x}_{4}=x_{4}\left(1-r^{2}\right)-\alpha_{1}\left(x_{3}^{2}-x_{1}^{2}-x_{2}^{2}\right)-\alpha_{2} x_{4}\left(x_{1}^{2}+x_{2}^{2}+x_{3}^{2}\right)-\lambda_{1} x_{1} x_{2} x_{3}
\end{array}\right.
$$

where

$$
r^{2}=x_{1}^{2}+x_{2}^{2}+x_{3}^{2}+x_{4}^{2}, \quad \alpha_{2}<0<\alpha_{1} \quad \text { and } \quad \alpha_{1}+\alpha_{2}>0
$$

The unit sphere $\mathbb{S}^{3} \subset \mathbb{R}^{4}$ is invariant under the corresponding flow and every trajectory with nonzero initial condition is forward asymptotic to it. Indeed, $\left\langle f_{(0,0)}(x), x\right\rangle<0$ for every $x \in \mathbb{S}^{3}$, where $\langle.,$.$\rangle denotes$ the usual inner product. Moreover, the origin is repelling since all eigenvalues of $D f_{(0,0)}$ at the origin have positive real part.

The vector field $f_{(0,0)}$ is equivariant under the action of the compact Lie group $\mathbb{Z}_{2}\left(\Gamma_{1}\right) \oplus \mathbb{Z}_{2}\left(\Gamma_{2}\right)$, where $\mathbb{Z}_{2}\left(\Gamma_{1}\right)$ and $\mathbb{Z}_{2}\left(\Gamma_{2}\right)$ act as

$$
\Gamma_{1}\left(x_{1}, x_{2}, x_{3}, x_{4}\right)=\left(-x_{1},-x_{2}, x_{3}, x_{4}\right) \quad \text { and } \quad \Gamma_{2}\left(x_{1}, x_{2}, x_{3}, x_{4}\right)=\left(x_{1}, x_{2},-x_{3}, x_{4}\right) .
$$

By construction, $\lambda_{1}$ is the controlling parameter of the $\mathbb{Z}_{2}\left(\Gamma_{2}\right)$-symmetry breaking and $\lambda_{2}$ controls the $\mathbb{Z}_{2}\left(\Gamma_{1}\right)$-symmetry.

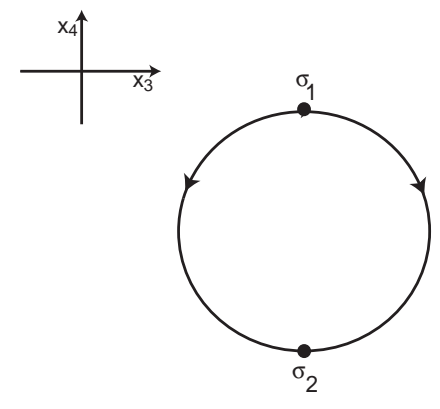

$\mathrm{x}_{1}=\mathrm{x}_{2}=0$

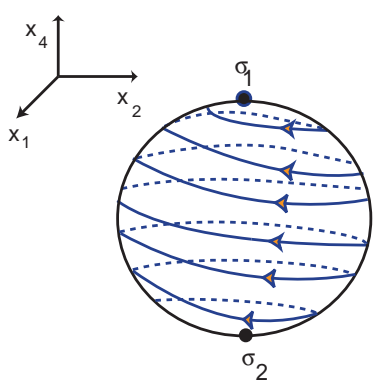

$x_{3}=0$

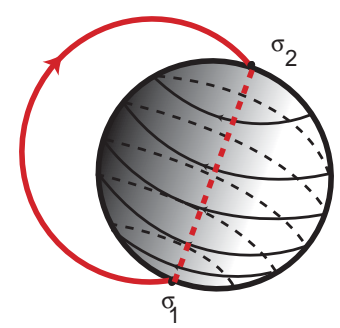

$\sigma_{1}$

Fig. 3. Heteroclinic connections of the organizing center when $\lambda_{1}=\lambda_{2}=0, \alpha_{1}=1$ and $\alpha_{2}=-0.1$.

For every $\lambda_{1}, \lambda_{2} \in[0,1]$, the flow of $f_{\left(\lambda_{1}, \lambda_{2}\right)}$ has two equilibria, namely

$$
\sigma_{1}=(0,0,0,+1) \quad \text { and } \quad \sigma_{2}=(0,0,0,-1)
$$

which are hyperbolic saddle-foci with different Morse indices (these indices are the dimensions of the unstable manifolds). The linearization of $f_{(0,0)}$ at $\sigma_{1}$ and $\sigma_{2}$ has eigenvalues

$$
-C_{1}=\left(\alpha_{2}-\alpha_{1}\right) \pm i, E_{1}=\alpha_{2}+\alpha_{1} \quad \text { and } \quad E_{2}=\left(\alpha_{2}+\alpha_{1}\right) \pm i,-C_{2}=\alpha_{2}-\alpha_{1},
$$

respectively. The selection of the values of $\alpha_{1}$ and $\alpha_{2}$ ensures that $\sigma_{1}$ and $\sigma_{2}$ are indeed saddle-foci.

As depicted in Figure 3, in these coordinates

$$
W_{(0,0)}^{u}\left(\sigma_{2}\right)=W_{(0,0)}^{s}\left(\sigma_{1}\right)=\left\{\left(x_{1}, x_{2}, x_{3}, x_{4}\right): x_{1}^{2}+x_{2}^{2}+x_{4}^{2}=1, x_{3}=0\right\}
$$

and the one-dimensional connection is the curve

$$
W_{(0,0)}^{u}\left(\sigma_{1}\right)=W_{(0,0)}^{s}\left(\sigma_{2}\right)=\left\{\left(x_{1}, x_{2}, x_{3}, x_{4}\right): x_{1}=0, x_{2}=0, x_{3}^{2}+x_{4}^{2}=1\right\} .
$$


These invariant manifolds of $\sigma_{1}$ and $\sigma_{2}$ lie inside the invariant circle $\operatorname{Fix}\left(\mathbb{Z}_{2}\left(\Gamma_{1}\right)\right) \cap \mathbb{S}^{3}$. Moreover, the two-dimensional invariant manifolds are contained in the invariant two-sphere $\operatorname{Fix}\left(\mathbb{Z}_{2}\left(\Gamma_{2}\right)\right) \cap \mathbb{S}^{3}$. It is precisely this symmetry that forces the two-invariant manifolds $W_{(0,0)}^{u}\left(\sigma_{2}\right)$ and $W_{(0,0)}^{s}\left(\sigma_{1}\right)$ to coincide. In what follows, we denote by $\Sigma_{0}$ the heteroclinic network formed by the two connections $\left[\sigma_{1} \rightarrow \sigma_{2}\right]$ and the sphere $\left[\sigma_{2} \rightarrow \sigma_{1}\right]$ (see Figure 3 ).

There are two possibilities for the geometry of the flow around each saddle-focus of the network $\Sigma_{0}$, depending on the direction the solutions turn around $\left[\sigma_{1} \rightarrow \sigma_{2}\right]$. Proposition 1 of [Rodrigues \& Labouriau, 2014] shows that, keeping $\lambda_{2}=0$, the equilibria $\sigma_{1}$ and $\sigma_{2}$ have the same chirality for every parameter value $\lambda_{1}$ under consideration. Moreover, this is a robust property as long as the one-dimensional heteroclinic connection persists.

Two comments are in order concerning the parameters. We will study approximations of the bifurcation diagram of the family $f_{\left(\lambda_{1}, \lambda_{2}\right)}$ in the space of the two parameters $\left(\lambda_{1}, \lambda_{2}\right)$, based on the signs of the Lyapunov exponents. We will restrict to the vector fields (1) with $\alpha_{1}=1$ and $\alpha_{2}=-0.1$. These systems have already been analyzed using other parameters. For instance, assuming transversality between the stable manifold of $\sigma_{2}$ and the unstable manifold of $\sigma_{1}$ (while $\lambda_{1}>0$ is fixed), Bykov uses in [Bykov, 2000] two parameters $\lambda_{2}$ and $\lambda_{3}$ to describe the position of the point of intersection of the stable manifold of $\sigma_{1}$ with the top of a linearization cylinder at $\sigma_{2}$ (see Figure 6). We note that breaking the 1-dimensional connection is a phenomenon of a codimension 2, so we should consider two parameters. However, due to the symmetry constraints, for the same phenomena we employ just one parameter, namely $\lambda_{2}$, since we have $\lambda_{3}=0$. Therefore, we expect differences between our bifurcation diagram and the one obtained in [Bykov, 2000]. For future comparison, we include in Figure 4 an illustration of the bifurcation diagram suggested in [Bykov, 1980]. To relate several parameters selected by other authors, we refer the reader to Table 1.

\begin{tabular}{|c|c|c|c|c|}
\hline & \multicolumn{4}{|c|}{ Reference } \\
\hline \hline Parameter & [Bykov, 2000, 1980] & [Algaba et al., 2010, 2011] & [Barrio et al., 2011] & This paper \\
\hline \hline$\lambda_{1}$ & $>0$ fixed & TP-line $(\mu<0)$ & not applicable & $\geq 0$ \\
\hline$\lambda_{2}$ & $\mu_{1}$ & $d_{1}$ & a & $\geq 0$ \\
\hline$\lambda_{3}$ & $\mu_{2}$ & $d_{2}$ & $c$ & 0 \\
\hline
\end{tabular}

\section{Glossary}

In this section, we introduce some terminology for vector fields acting in three-dimensional Riemannian manifolds that we will use in the remaining sections.

Lyapunov exponents: Given a vector field on a $n$-dimensional manifold, its Lyapunov exponents are related to the expanding or contracting nature of different directions in the phase space. To estimate them, one monitors the long-term evolution of an infinitesimal $n$-sphere of initial conditions according to the algorithm of [Wolf et al., 1985]. More precisely, this $n$-sphere becomes an $n$-ellipsoid due to the locally deforming nature of the flow; then, the $i^{t h}$ one-dimensional Lyapunov exponent is defined in terms of the length of the ellipsoidal principal axis $p_{i}(t)$ as follows:

$$
\lambda_{i}=\lim _{t \rightarrow+\infty} \frac{1}{t} \log _{2} \frac{p_{i}(t)}{p_{i}(0)}
$$


Chaotic attracting set: An invariant set which contains the $\omega$-limit of all the points in one of its neighborhoods and exhibits one or more positive Lyapunov exponents.

Shilnikov cycle: A homoclinic connection of an equilibrium which is a saddle-focus (that is, a zero of a vector field whose linearization has a pair of complex eigenvalues). The dynamics near this cycle may be either attracting or chaotic depending on the ratio between the expanding and contracting eigenvalues.

Bykov attractor: An attracting heteroclinic cycle between two hyperbolic saddle-foci of different Morse indices whose invariant manifolds coincide.

Bykov cycle: A heteroclinic cycle between two hyperbolic saddle-foci equilibria of different Morse indices, where one of the connections is transversal (and so stable under small perturbations) while the other is structurally unstable.

Bykov network: A connected union of heteroclinic cycles, not necessarily in finite number.

T-point (Terminal point): A point in the parameter space for which we observe a Bykov cycle in the phase diagram. In three-dimensional systems, $T$-points are codimension-two organizing centers of global bifurcations and complex behavior.

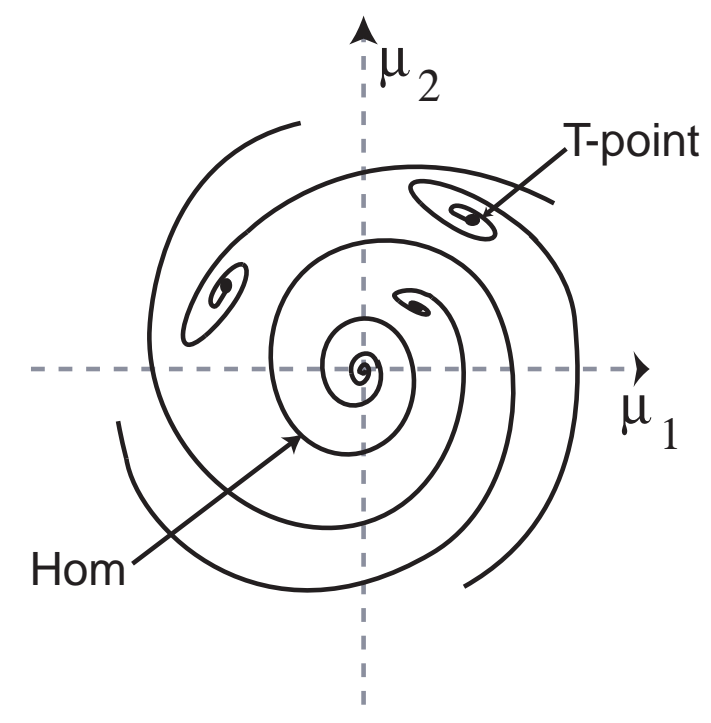

Fig. 4. Sketch of a partial unfolding of a $T$-point of codimension-two corresponding to a heteroclinic connection between two saddle-foci. It includes two spiralling bifurcation curves, each one corresponding to a homoclinic bifurcation of a saddle-focus (for each $T$-point, though only one is depicted) inspired by [Bykov, 2000; Homburg \& Sandstede, 2010]. The number of loops of the separatrix around the saddle-focus increases with each turn of the spiral. Each extra tip point $\left(\right.$ besides $\left.\left(\mu_{1}, \mu_{2}\right)=(0,0)\right)$ corresponds to a subsidiary $T$-point.

B-point (Belyakov point): A point in the parameter space for which the eigenvalues of one of the equilibria change from complex to real.

Shrimp: A region in the bifurcation diagram with a peculiar form, for whose parameters the chosen orbit is attracted by a superstable sink. Such a region exhibits several fold bifurcation lines and cusp bifurcation points. More details in [Barrio et al., 2012; Gallas, 2010].

\section{Analysis near the organizing center $\lambda_{1}=\lambda_{2}=0$}

In this section we establish local coordinates near the saddle-foci $\sigma_{1}$ and $\sigma_{2}$. The starting point is an application of the Linearization Theorem of [Deng, 1993] to describe the flow around the equilibria, using cylindrical coordinates in neighborhoods of each saddle-focus whose boundaries are transverse to the 
linearized flow. Thereby, for each equilibrium we obtain the expression of the local map that sends points in the boundary where the flow goes into points in the boundary where the flow goes out. Finally, we introduce a model for the transition maps from one of the previous neighborhoods to the other.

\subsection{Local coordinates}

In order to describe the dynamics in a neighborhood of the Bykov attractor $\Sigma_{0}$, we need a workable expression of the Poincaré map of $f_{\left(\lambda_{1}, \lambda_{2}\right)}(x) \equiv f\left(x, \lambda_{1}, \lambda_{2}\right)$ at a suitable cross section inside this neighborhood. In the linearizing coordinates coordinates, after a re-scaling of the variables, we consider two cylindrical neighborhoods $V_{\sigma_{1}}$ and $V_{\sigma_{2}}$ in $\mathbb{S}^{3}$ of $\sigma_{1}$ and $\sigma_{2}$, respectively, with base-radius 1 and height 2 . The boundaries of $V_{\sigma_{1}}$ and $V_{\sigma_{2}}$ have three components (see Figure 5):

(1) The cylinder wall, parameterized in cylindrical coordinates $(\rho, \theta, z)$ by

$$
\theta \in[0,2 \pi],|z| \leq 1 \quad \mapsto \quad(1, \theta, z) .
$$

(2) Two discs, the top and bottom of each cylinder, parameterized by

$$
\rho \in[0,1], \theta \in[0,2 \pi] \quad \mapsto \quad(\rho, \theta, \pm 1) .
$$

Observe that the local stable manifold $W_{\text {loc }}^{s}\left(\sigma_{1}\right)$ of the equilibrium $\sigma_{1}$ is the disk in $V_{\sigma_{1}}$ given by $\{(\rho, \theta, z): 0 \leq \rho \leq 1,0 \leq \theta \leq 2 \pi, z=0\}$. The local stable manifold $W_{\mathrm{loc}}^{s}\left(\sigma_{2}\right)$ of $\sigma_{2}$ is the $z$-axis in $V_{\sigma_{2}}$; the local unstable manifold $W_{\mathrm{loc}}^{u}\left(\sigma_{2}\right)$ of $\sigma_{2}$ is parameterized by $z=0$ in $V_{\sigma_{2}}$.
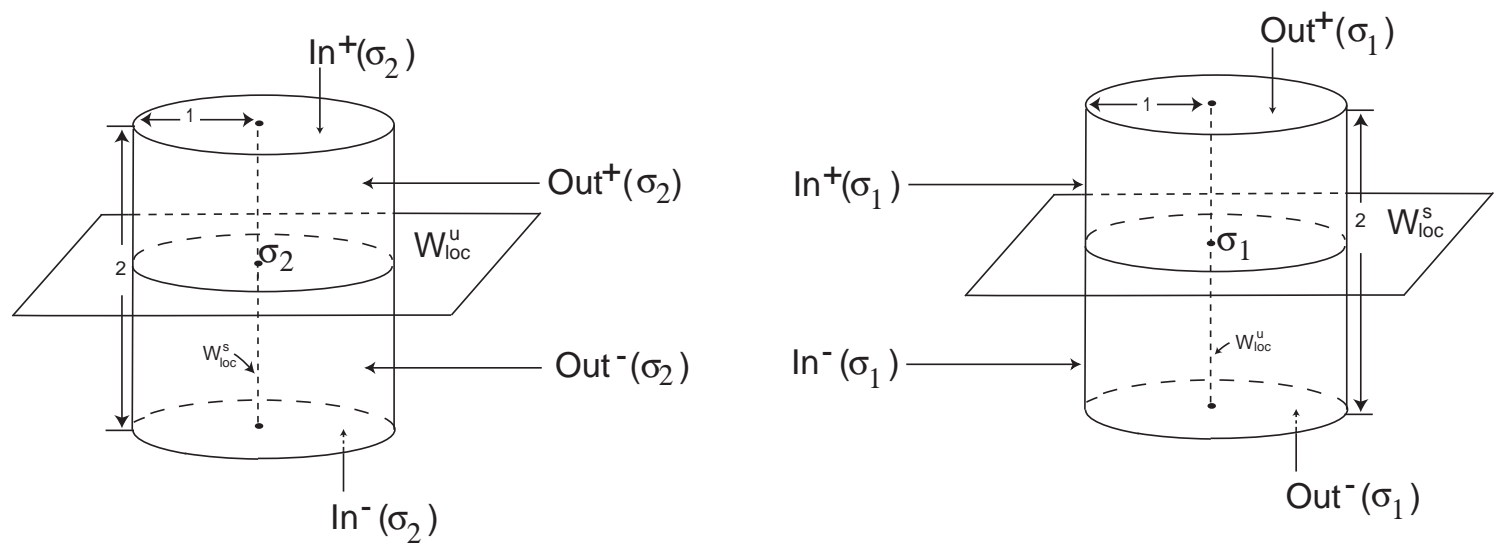

Fig. 5. Local coordinates near $\sigma_{1}$ and $\sigma_{2}$.

In $V_{\sigma_{1}}$, we will use the following notation:

$$
\begin{aligned}
\operatorname{Out}^{+}\left(\sigma_{1}\right):= & \text { top of } V_{\sigma_{1}} \text {, that is, }\{(\rho, \theta, 1): 0 \leq \rho<1, \theta \in[0,2 \pi]\} . \\
\text { Out }^{-}\left(\sigma_{1}\right):= & \text { bottom of } V_{\sigma_{1}} \text {, that is, }\{(\rho, \theta,-1): 0 \leq \rho<1, \theta \in[0,2 \pi]\} . \\
\operatorname{In}^{+}\left(\sigma_{1}\right):= & \text { upper part of the cylinder wall of } V_{\sigma_{1}}, \text { that is, the set } \\
& \{(1, \theta, z): \theta \in[0,2 \pi], 0<z<1\} . \\
\operatorname{In}^{-}\left(\sigma_{1}\right):= & \text { lower part of the cylinder wall of } V_{\sigma_{1}} \text {, that is, the set } \\
& \{(1, \theta, z): \theta \in[0,2 \pi],-1<z<0\} . \\
\operatorname{In}\left(\sigma_{1}\right):= & \operatorname{In}^{+}\left(\sigma_{1}\right) \cup \operatorname{In}^{-}\left(\sigma_{1}\right) ; \text { its elements enter } V_{\sigma_{1}} \text { after a positive time. } \\
\text { Out }\left(\sigma_{1}\right):= & \operatorname{Out}^{+}\left(\sigma_{1}\right) \cup \operatorname{Out}^{-}\left(\sigma_{1}\right) ; \text { its elements leave } V_{\sigma_{1}} \text { after a positive time. }
\end{aligned}
$$

By construction, the flow is transverse to these sections. Similarly, we define the cross sections for the linearization around $\sigma_{2}$. We will refer to $\operatorname{In}\left(\sigma_{2}\right)$, the top and the bottom of $V_{\sigma_{2}}$, consisting of points that 
enter $V_{\sigma_{2}}$ in positive time; Out $\left(\sigma_{2}\right)$, the cylinder wall of $V_{\sigma_{2}}$, made of points that go inside $V_{\sigma_{2}}$ in negative time, while $\mathrm{Out}^{+}\left(\sigma_{2}\right)$ denotes its upper part and $\mathrm{Out}^{-}\left(\sigma_{2}\right)$ its lower part. Notice that $\left[\sigma_{1} \rightarrow \sigma_{2}\right]$ connects points with $z>0$ in $V_{\sigma_{1}}$ (respectively $z<0$ ) to points with $z>0$ (respectively $z<0$ ) in $V_{\sigma_{2}}$.

\subsection{Poincaré maps near the saddle-foci}

As the dynamics sends points with $z>0$ in $V_{\sigma_{1}}$ (respectively $z<0$ ) to points with $z>0$ (respectively $z<0$ ) in $V_{\sigma_{2}}$, and is symmetric with respect to the plane $z=0$ (which is Fix $\left(\Gamma_{2}\right) \cap \mathbb{S}^{3}$ ), it is enough to analyze the orbits of initial conditions $(\rho, \theta, z)$ in the invariant upper part $z>0$.

In cylindrical coordinates $(\rho, \theta, z)$, the linearization of the dynamics at $\sigma_{1}$ and $\sigma_{2}$ is specified by the following equations:

$$
\left\{\begin{array} { l } 
{ \dot { \rho } = - C _ { 1 } \rho } \\
{ \dot { \theta } = \omega _ { 1 } } \\
{ \dot { z } = E _ { 1 } z }
\end{array} \quad \text { and } \quad \left\{\begin{array}{l}
\dot{\rho}=E_{2} \rho \\
\dot{\theta}=\omega_{2} \\
\dot{z}=-C_{2} z
\end{array}\right.\right.
$$

Therefore, a trajectory whose initial condition is $(1, \theta, z) \in \mathrm{In}^{+}\left(\sigma_{1}\right)$ arrives at $\mathrm{Out}^{+}\left(\sigma_{1}\right)$ after a period of time equal to

$$
-\frac{\log z}{E_{1}}
$$

Moreover, the trajectory of such a point $(1, \theta, z)$ leaves $V_{\sigma_{1}}$ through $\operatorname{Out}^{+}\left(\sigma_{1}\right)$ at the point of $\operatorname{Out}^{+}\left(\sigma_{1}\right)$ given by

$$
\Phi_{1}^{+}(1, \theta, z)=\left(z^{\delta_{1}}+\mathcal{S}_{1}(1, \theta, z),\left[-\frac{\omega_{1}}{E_{1}} \log z+\theta+\mathcal{S}_{2}(1, \theta, z)\right] \bmod 2 \pi, 1\right)
$$

where $\mathcal{S}_{1}$ and $\mathcal{S}_{2}$ are smooth functions such that, for $i=1,2$ and every $k, \ell \in \mathbb{N}_{0}$,

$$
\left|\frac{\partial^{k+\ell} \mathcal{S}_{i}(1, \theta, z)}{\partial \theta^{k} \partial z^{\ell}}\right|=\mathcal{O}\left(z^{\delta_{1}(1+\varepsilon)}\right)
$$

for some constant $0<\varepsilon<1$. Dually, a point $(\rho, \theta, 1) \in \operatorname{In}^{+}\left(\sigma_{2}\right)$ leaves $V_{\sigma_{2}}$ through $\operatorname{Out}^{+}\left(\sigma_{2}\right)$ after a period of time equal to

$$
-\frac{\log \rho}{E_{2}}
$$

at the point of $\mathrm{Out}^{+}\left(\sigma_{2}\right)$ defined by

$$
\Phi_{2}^{+}(\rho, \theta, 1)=\left(1,\left[-\frac{\omega_{2}}{E_{2}} \log \rho+\theta+\mathcal{T}_{1}(\rho, \theta, 1)\right] \bmod 2 \pi, \rho^{\delta_{2}}+\mathcal{T}_{2}(\rho, \theta, 1)\right)
$$

where $\mathcal{T}_{1}$ and $\mathcal{T}_{2}$ satisfy a condition similar to (4). The maps $\mathcal{S}_{1}, \mathcal{S}_{2}, \mathcal{T}_{1}$ and $\mathcal{T}_{2}$ represent asymptotically small terms which approach zero when either $\rho$ or $z$ goes to zero. With this choice of local coordinates, the $\operatorname{maps} \Phi_{1}^{ \pm}$and $\Phi_{2}^{ \pm}$do not depend on $\lambda_{1}$ nor on $\lambda_{2}$.

\subsection{The first transition}

Points in $\mathrm{Out}^{+}\left(\sigma_{1}\right)$ near $W^{u}\left(\sigma_{1}\right)$ are mapped into $\operatorname{In}^{+}\left(\sigma_{2}\right)$ along a flow-box around the connection $\left[\sigma_{1} \rightarrow \sigma_{2}\right]$. Up to a change of coordinates, corresponding to homotheties and rotations which leave invariant the local expressions of the flows in neighborhoods of the equilibria, we may assume that, for $\lambda_{2}>0$, the transition map

$$
\Psi_{1 \rightarrow 2}^{+}: \operatorname{Out}^{+}\left(\sigma_{1}\right) \rightarrow \operatorname{In}^{+}\left(\sigma_{2}\right)
$$

is an affine map given, in rectangular coordinates $x=\rho \cos \theta, y=\rho \sin \theta$ and $z$, by the composition $L_{1} \circ L_{2}$, where

$$
L_{1}(x, y, 1)=\left(\begin{array}{l}
x \\
y \\
1
\end{array}\right)+\left(\begin{array}{c}
\lambda_{2} \\
0 \\
1
\end{array}\right) \quad \text { and } \quad L_{2}(x, y, 1)=\left(\begin{array}{ccc}
a & 0 & 0 \\
0 & \frac{1}{a} & 0 \\
0 & 0 & 1
\end{array}\right)\left(\begin{array}{l}
x \\
y \\
1
\end{array}\right)
$$


for some constant $\left.\left.a=a\left(\lambda_{1}, \lambda_{2}\right) \in\right] 0,1\right]$, taking modulus $2 \pi$ on the variable $\theta$. A similar transition will be assumed acting from $\mathrm{Out}^{-}\left(\sigma_{1}\right)$ to $\mathrm{In}^{-}\left(\sigma_{2}\right)$. Observe that, to compose this map with $\Phi_{2}$, it is necessary to go back to cylindrical coordinates.

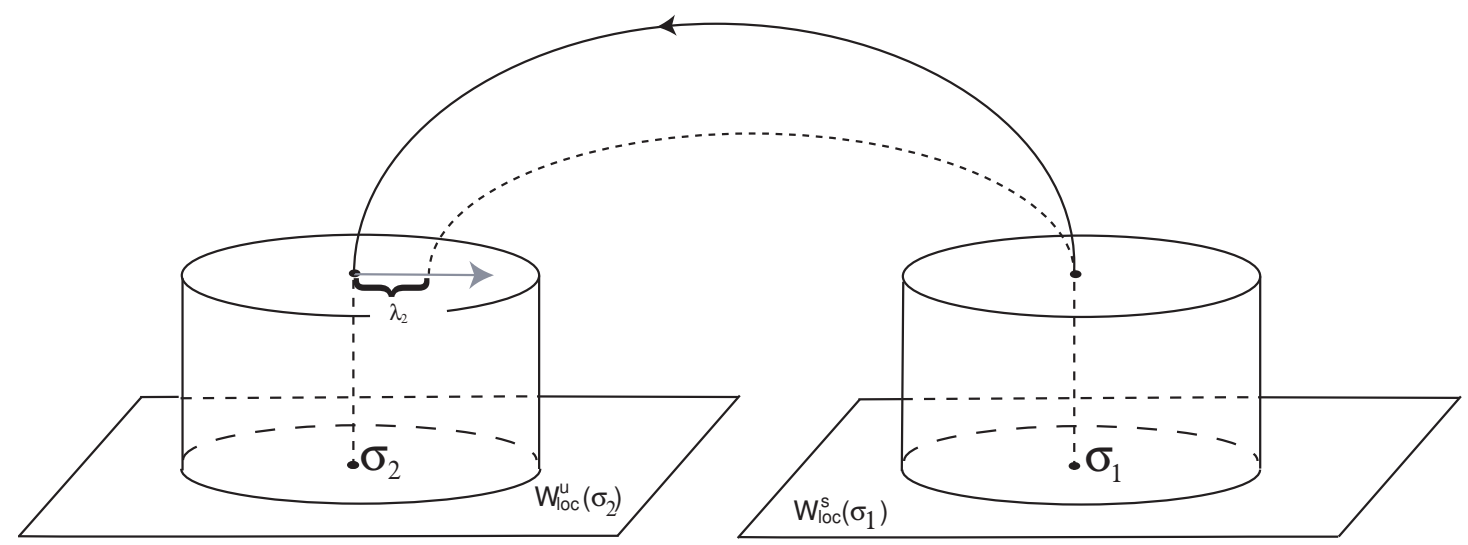

Fig. 6. The effect of perturbation that breaks the $\mathbb{Z}_{2}\left(\Gamma_{1}\right)$-symmetry.

\subsection{The second transition}

When $\lambda_{1}>0$ is small, the local and global maps will change slightly but not significantly. We still have a well defined transition map

$$
\Psi_{2 \rightarrow 1}: \operatorname{Out}\left(\sigma_{2}\right) \rightarrow \operatorname{In}\left(\sigma_{1}\right)
$$

that depends on the $\mathbb{Z}_{2}\left(\Gamma_{2}\right)$-symmetry breaking parameter $\lambda_{1}$. We are assuming that $W^{s}\left(\sigma_{1}\right)$ intersects the wall $\operatorname{Out}\left(\sigma_{2}\right)$ of the cylinder $V_{\sigma_{2}}$ along an ellipse, an event compatible with the expected unfolding from the coincidence of the invariant manifolds of the two equilibria we observe when $\lambda_{1}=0=\lambda_{2}$. Accordingly, we will suppose that the map

$$
\Psi_{2 \rightarrow 1}: \operatorname{Out}\left(\sigma_{2}\right) \longrightarrow \operatorname{In}\left(\sigma_{1}\right)
$$

is given, in cylindrical coordinates, by

$$
\Psi_{2 \rightarrow 1}(1, \theta, z)=\left(1, \theta, z+\lambda_{1} \sin \theta\right) .
$$

Notice that the transition map $\Psi_{2 \rightarrow 1}$ depends on $\lambda_{1}$ but not on $\lambda_{2}$. Similarly, the other transition map $\Psi_{1 \rightarrow 2}$ depends on $\lambda_{2}$ but not on $\lambda_{1}$.

On the sets $W^{u}\left(\sigma_{2}\right) \cap \operatorname{In}\left(\sigma_{1}\right)$ and $W^{s}\left(\sigma_{1}\right) \cap \operatorname{Out}\left(\sigma_{2}\right)$, which are described by the curve

$$
\theta \mapsto\left(1, \theta, \lambda_{1} \sin \theta\right)
$$

we get

$$
\Phi_{1}\left(1, \theta, \lambda_{1} \sin \theta\right)=\left(\left(\lambda_{1} \sin \theta\right)^{\delta_{1}},-\frac{\omega_{1}}{E_{1}} \ln \left(\lambda_{1} \sin \theta\right)+\theta, 1\right)+\text { h.o.t. }
$$

and

$$
\Phi_{2}^{-1}\left(1, \theta, \lambda_{1} \sin \theta\right)=\left(\left(\lambda_{1} \sin \theta\right)^{\frac{1}{\delta_{2}}}, \frac{\omega_{2}}{C_{2}} \ln \left(\lambda_{1} \sin \theta\right)+\theta, 1\right)+\text { h.o.t. }
$$

Therefore, as depicted in Figure 7, we conclude that: 


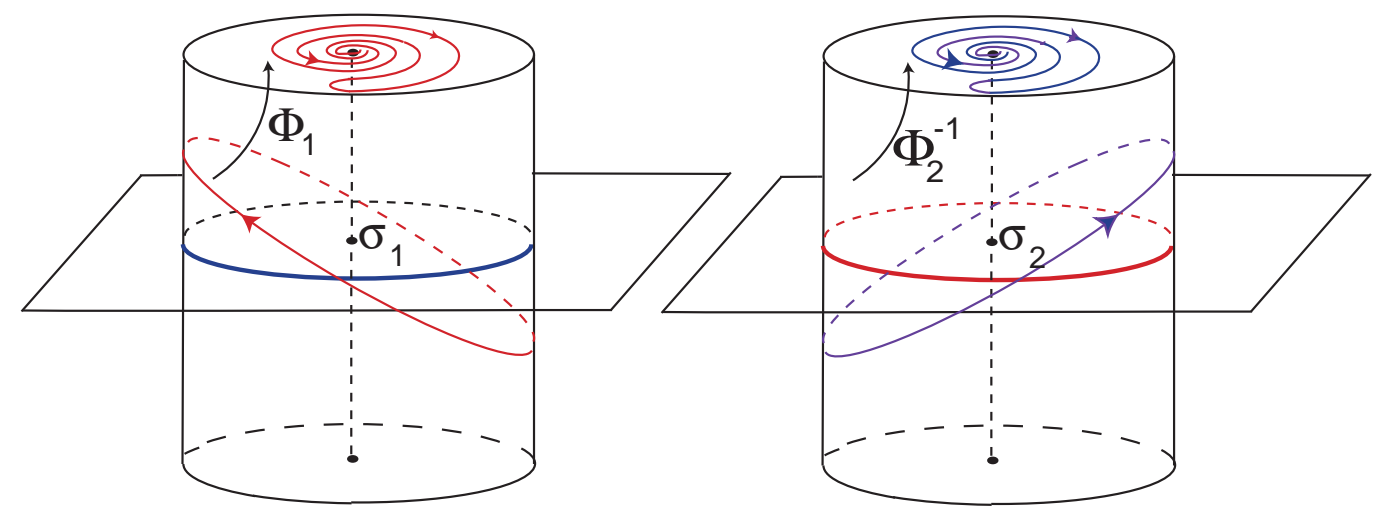

Fig. 7. The double spirals of Lemma 1.

Lemma 1. [Labouriau \& Rodrigues, 2016] For $\theta \in] \pi / 2,3 \pi / 2[$, the sets

$$
\left\{\Phi_{1}\left(1, \theta, \lambda_{1} \sin (\theta)\right): \theta \in\right] \pi / 2,3 \pi / 2[\} \quad \text { and } \quad\left\{\Phi_{2}^{-1}\left(1, \theta, \lambda_{1} \sin (\theta)\right): \theta \in\right] \pi / 2,3 \pi / 2[\}
$$

are double spirals on Out $\left(\sigma_{1}\right)$ and $\operatorname{In}\left(\sigma_{2}\right)$ accumulating respectively on $W^{u}\left(\sigma_{1}\right) \cap$ Out $\left(\sigma_{1}\right)$ and $W^{s}\left(\sigma_{2}\right) \cap$ $\operatorname{In}\left(\sigma_{2}\right)$.

The next table summarizes some of the information of the three previous subsections.

\begin{tabular}{|c|c|c|c|}
\hline Parameters & Symmetries preserved & $\Psi_{1 \rightarrow 2}$ & $\Psi_{2 \rightarrow 1}$ \\
\hline \hline$\lambda_{1}=\lambda_{2}=0$ & $\Gamma_{1}, \Gamma_{2}$ & $L_{2}$ & Identity \\
\hline$\lambda_{1} \neq 0$ and $\lambda_{2}=0$ & $\Gamma_{1}$ & $L_{2}$ & Model (6) \\
\hline$\lambda_{1}=0$ and $\lambda_{2} \neq 0$ & $\Gamma_{2}$ & $L_{1} \circ L_{2}$ & Identity \\
\hline$\lambda_{1} \neq 0$ and $\lambda_{2} \neq 0$ & Identity & $L_{1} \circ L_{2}$ & Model $(6)$ \\
\hline
\end{tabular}

\section{The selection of initial conditions}

Since the invariant manifolds of the equilibria play an essential role in the unfolding of the heteroclinic cycle, the following choice of the initial conditions $\left(x_{1}, x_{2}, x_{3}, x_{4}\right) \in \mathbb{S}^{3}$ aims to grasp the main dynamical properties. The position of some of them on the phase space is indicated on Figure 10. Our selection is the following:

CI1 An initial condition lying in the local unstable manifold of $\sigma_{1}$, as $(0,0,0.01,0.9999)$. 
CI2 An initial condition near the 1D-heteroclinic connection, such as $(0.01,0.01,0.01,1)$.

CI3 An initial condition lying in the local unstable manifold of $\sigma_{2}$, such as $(0.01,0.01,0,-1)$.

CI4, CI5 The initial conditions $(-0.5,-0.129,-0.8807,-0.3013)$ and $(0,0,1,0.1)$ used to illustrate multistability behaviour.

\section{The code of colors}

Although the system we study lives in $\mathbb{R}^{4}$, the analysis may be performed in the unit three-dimensional sphere $\mathbb{S}^{3}$ since it is globally attracting (cf. Section 2). Depending on the control parameters of the system, different dynamical regimes have been identified both analytically and numerically, namely quasi-periodic motions, homoclinic and heteroclinic connections and transitions to chaotic oscillations. According to [Wolf et al., 1985], for a three-dimensional continuous dissipative flow, the only possible spectra and the attractors they describe are as follows:

$$
\begin{aligned}
(-,-,-) & \rightarrow \text { the } \omega \text {-limit of the corresponding orbit contains an equilibrium point. } \\
(0,-,-) & \rightarrow \text { the } \omega \text {-limit of the corresponding orbit is a periodic sink. } \\
(+, 0,-) & \rightarrow \text { the } \omega \text {-limit of the corresponding orbit is a chaotic attractor. } \\
(0,0,-) & \rightarrow \text { the } \omega \text {-limit of the corresponding orbit is a } 2 \text {-torus attractor. }
\end{aligned}
$$

The bifurcation diagrams we will discuss have been drawn by two different methods. The parameter plane is scanned with a sufficiently small step along each coordinate axes, and meanwhile:

Figure 20 The software evaluates at each parameter value how many Lyapunov exponents along the orbit with initial condition CI1 are positive. Afterwards, the parameter is painted according to the following rules: red for 0 , blue for 1 , yellow for 2 .

Figures 2, 16, 21, 22, 23 For each parameter value, the program colors the point in yellow-red scale (see the colorbar on each figure) if the first (maximum) Lyapunov exponent is positive for the considered trajectory, otherwise (first Lyapunov exponent equal to zero) the point is colored in grey scale (darker or lighter) using the second Lyapunov exponent. To estimate the complete Lyapunov spectra we use the well known algorithm for differential equations introduced in [Wolf et al., 1985] with a Taylor series integrator [Abad et al., 2012].

\section{7. $\lambda_{1}=0$ and $\lambda_{2}=0$}

This is the starting vector field of the family we are considering, and its dynamics is quite simple: it has an attracting heteroclinic network between the two saddle-foci.

\subsection{Dynamics}

The two saddle-foci, together with their invariant manifolds, build a heteroclinic network $\Sigma_{0}$ which is asymptotically stable (that is, there exists an open neighborhood $V_{0}$ of $\Sigma_{0}$ such that every trajectory starting in $V_{0}$ remains in it for every positive time and is forward asymptotic to the network $\left.\Sigma_{0}\right)$. Indeed, the heteroclinic connections in $\Sigma_{0}$ are contained in fixed point subspaces satisfying the assumptions of [Krupa \& Melbourne, 1995]; hence, as the inequality $C_{1} C_{2}>E_{1} E_{2}$ holds, the stability criterion of [Krupa \& Melbourne, 1995] may be applied, ensuring that $\Sigma_{0}$ is asymptotically stable. This global attractor, so called Bykov attractor, has a finite modulus of stability [Carvalho \& Rodrigues, 2018] and the points of its proper basin of attraction have historic behavior [Carvalho \& Rodrigues, 2018].

The non-wandering set of the vector field $f_{(0,0)}$ is $\Sigma_{0}$, which contains the initial conditions CI1 and CI3. The coincidence of the two-dimensional invariant manifolds of $\sigma_{1}$ and $\sigma_{2}$ prevents visits to both cycles, and 


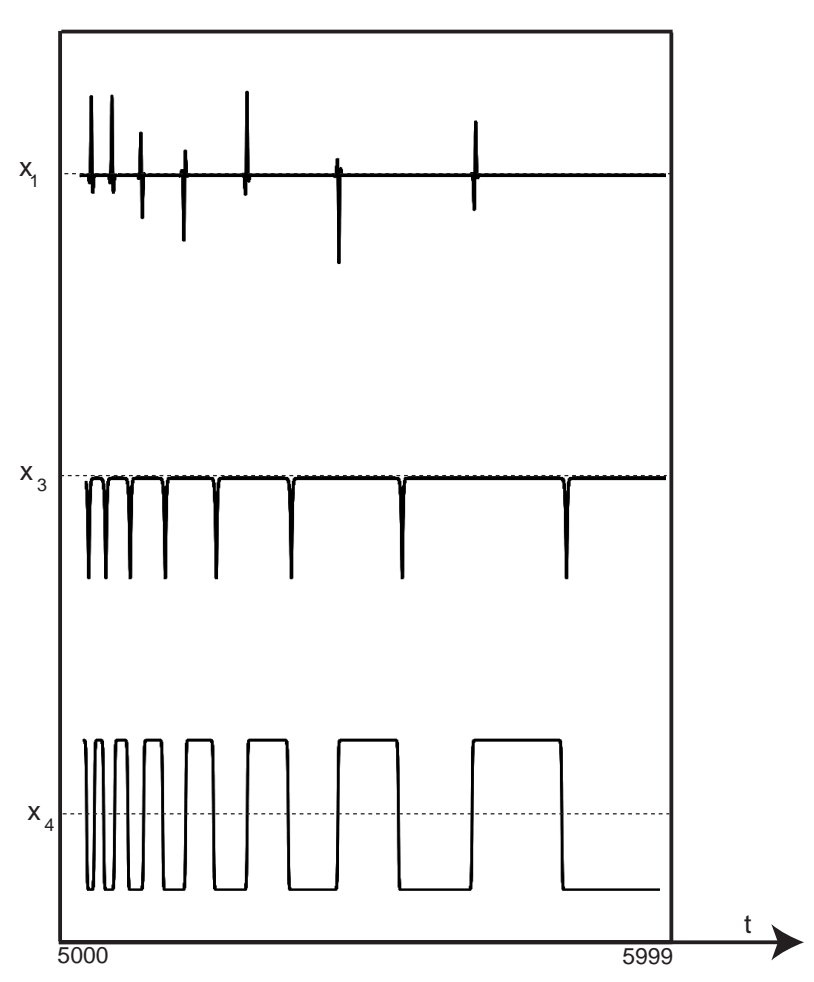

Fig. 8. Projection on the $x_{1}, x_{3}$ and $x_{4}$ coordinates of the solution of (1) using CI2, $\lambda_{1}=\lambda_{2}=0, \alpha_{1}=1$ and $\alpha_{2}=-0.1$.
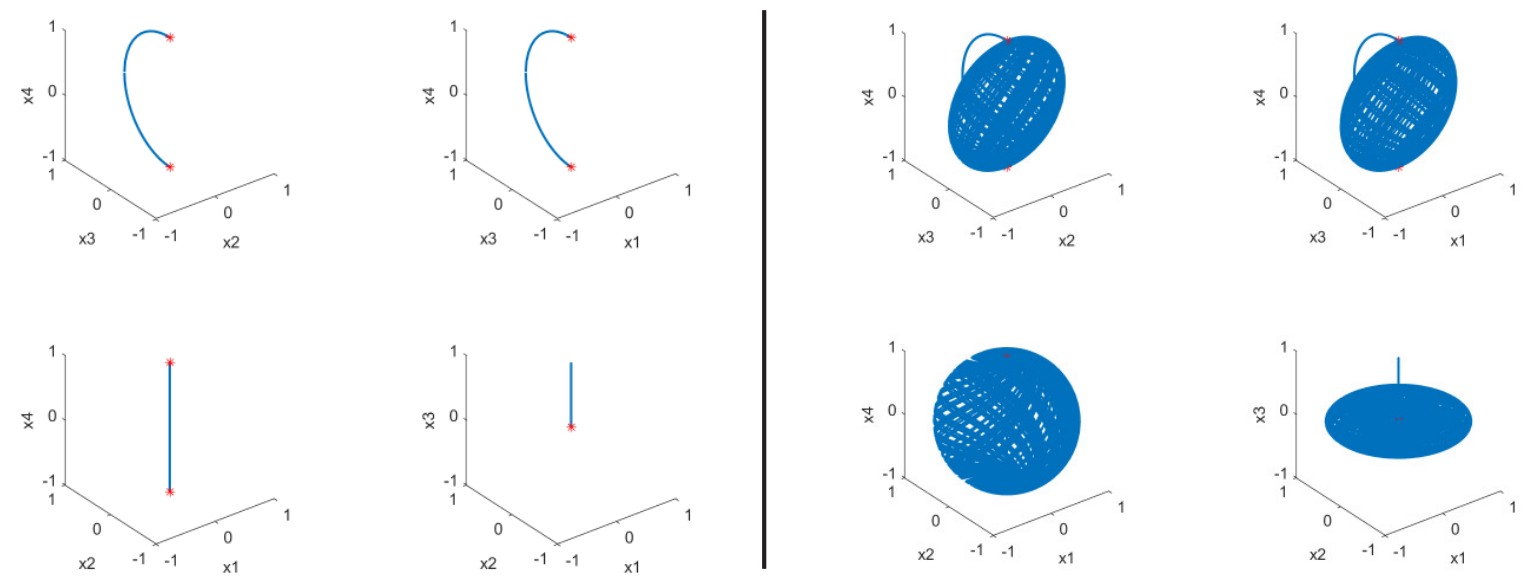

Fig. 9. Heteroclinic connections when $\lambda_{1}=\lambda_{2}=0, \alpha_{1}=1$ and $\alpha_{2}=-0.1$, realized by the orbit with initial condition CI1 (four images at left) and CI2 (right), with $t \in[0,10000]$.

that is why the $x_{3}$-component in Figure 8 has a constant sign. The geometric shape of the heteroclinic connections in $\Sigma_{0}$ is shown in Figure 9 and is consistent with the schemes of Figure 3.

\subsection{Bifurcation routes}

The two small parameters $\lambda_{1}$ and $\lambda_{2}$ control the symmetry-breaking which may be accomplished by two ways: either the stable manifold of $\sigma_{1}$ and the unstable manifold of $\sigma_{2}$ become transversal (when $\lambda_{1}>0$ and $\lambda_{2}=0$ ) or the one-dimensional connection is destroyed (when $\lambda_{1}=0$ and $\lambda_{2}>0$ ), or else these two phenomena occur simultaneously (when $\lambda_{1}>0$ and $\lambda_{2}>0$ ). See Figure 10.

In the next sections, we will discuss the dynamics and the possible bifurcations within these three 


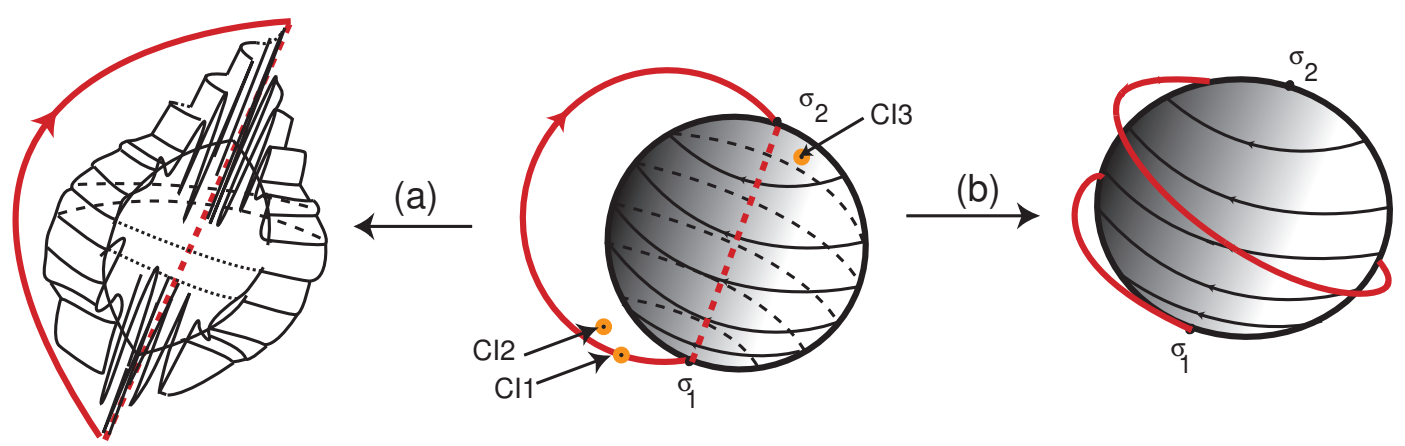

Fig. 10. Breaking: (a) the $\mathbb{Z}_{2}\left(\Gamma_{2}\right)$-symmetry and (b) the $\mathbb{Z}_{2}\left(\Gamma_{1}\right)$-symmetry.

scenarios based on numerical simulations and theoretical results.

\section{8. $\lambda_{1}>0$ and $\lambda_{2}=0$}

When the parameter $\lambda_{1}$ becomes positive, the vector field $f_{\left(\lambda_{1}, 0\right)}$ is no longer $\mathbb{Z}_{2}\left(\Gamma_{2}\right)$-equivariant, though it still has an attracting set. According to [Rodrigues \& Labouriau, 2014, Th. 2], the two-dimensional connection breaks into transverse intersections of the two-dimensional invariant manifolds, while, due to the remaining $\mathbb{Z}_{2}\left(\Gamma_{1}\right)$-symmetry, the one-dimensional connections are preserved. We will start describing the most relevant known features of the non-wandering set for small values of the parameter $\lambda_{1}>0$.

\subsection{Dynamics}

The heteroclinic connections in $\Sigma_{0}$ (see the central image of Figure 10) are contained in fixed point subspaces satisfying the assumptions of Krupa-Melbourne stability criterion [Krupa \& Melbourne, 1995]. Thus, there exists a three-dimensional normally hyperbolic invariant manifold whose boundary is transverse to the flow, diffeomorphic to a genus two handle-body, where every solution eventually enters. Therefore, there exists $\varepsilon_{0}>0$ such that, for $\left(\lambda_{1}, \lambda_{2}\right) \in\left[0, \varepsilon_{0}\right] \times\left[0, \varepsilon_{0}\right]$, the flow of $f_{\left(\lambda_{1}, \lambda_{2}\right)}$ has a three-dimensional normally hyperbolic invariant manifold, diffeomorphic to a genus two handle-body and transverse to the flow, which is inwardly oriented.

The main dynamical changes one observes when the parameter $\lambda_{1}$ becomes positive are due to the fact that now $W^{u}\left(\sigma_{2}\right)$ and $W^{s}\left(\sigma_{1}\right)$ intersect transversally. This gives rise to a countable union of saturated horseshoes, say $\Lambda_{\lambda_{1}}$, which accumulates on a network $\Sigma_{\lambda_{1}}$, as illustrated in Figures 10 (left) and 11. Since the basin of attraction of a $C^{2}$-uniformly hyperbolic horseshoe has Lebesgue measure zero, the set $\Lambda_{\lambda_{1}}$ is not directly observable, unless one finds a set with positive Lebesgue measure of initial conditions whose orbits have $\omega$-limits intersecting these horseshoes.

The transition from a simple steady state at $\lambda_{1}=0$ to a chaotic attractor with the increasing parameter $\lambda_{1}$ is an example of a family of vector fields exhibiting instant chaos, as the ones presented in [Guckenheimer \& Worfolk, 1992]. For instance, when $\lambda_{1}$ changes, one witnesses the creation and destruction of invariant pieces of the non-wandering set, as well as the loss of the hyperbolic properties at some of them. As illustrated in Figure 12, the transition maps undergo saddle-node and pitchfork bifurcations, besides cascades of period-doubling [Yorke \& Alligood, 1983] and the unfolding of homoclinic tangencies [Palis \& Takens, 1995] and Newhouse phenomena. Additionally, some of these bifurcations may happen simultaneously, while horseshoes and sinks vanish (cf. [Crovisier, 2002]).

The complex eigenvalues of the equilibria induce the spreading of solutions starting near either the unstable manifold of $\sigma_{2}$ or the stable manifold of $\sigma_{1}$, forcing visits to arbitrarily small neighborhoods of several homoclinic or heteroclinic connections of the equilibria. Meanwhile, homoclinic tangencies of periodic solutions exist in a countable subset of parameters $\lambda_{1}$ inside a small interval $] 0, \varepsilon_{1}[$, for some $\varepsilon_{1}>0$ [Labouriau \& Rodrigues, 2016], whose generic unfolding produces infinitely many sinks and possibly chaotic attractors. Those sinks seem to have very long periods and wander very close to $W^{u}\left(\sigma_{2}\right) \cup W^{s}\left(\sigma_{1}\right)$, 
(a)

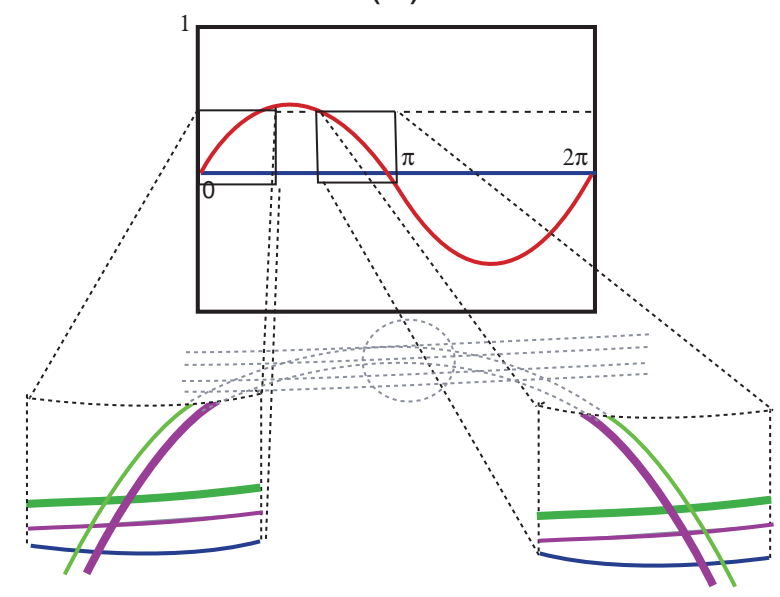

(b)

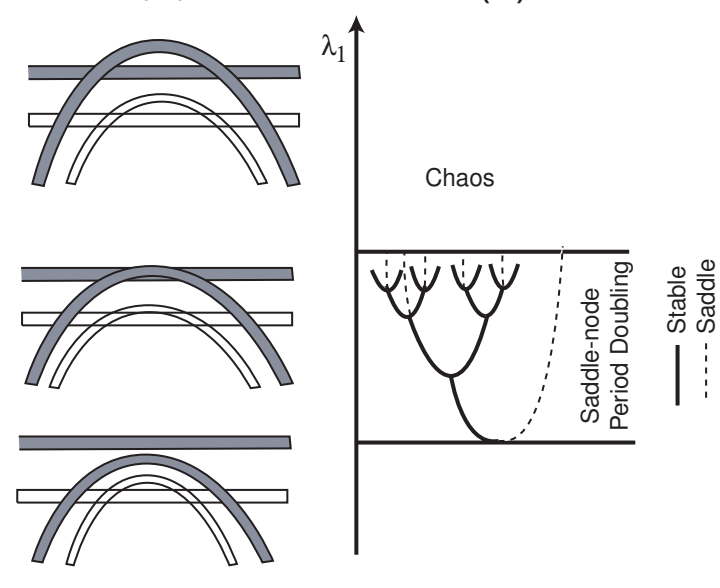

Fig. 11. Horseshoe destruction. (a) Configuration of the horseshoe in $\operatorname{In}\left(\sigma_{1}\right)$. The red sinusoidal curve, whose existence is assumed in the model (6), is precisely the red ellipse of Figure 7. (b) Geometric structure of the strips as $\lambda_{1}$ decreases. (c) Bifurcations.

spending large periods of time near the equilibria. They visit the tiny gaps of the horseshoes in $\Lambda_{\lambda_{1}}$, whose periodic trajectories somehow guide the sinks that appear nearby. In particular, every arbitrarily small neighborhood of the one-dimensional unstable manifold $W^{u}\left(\sigma_{1}\right)$ contains pieces of orbits of the horseshoes' saddles and of sinks, thus generating the so called heteroclinic switching [Aguiar et al., 2010]. Observe also that the two-dimensional invariant manifolds of the equilibria and the invariant manifolds of $\Lambda_{\lambda_{1}}$ also intersect, giving rise to a spiralling quasi-stochastic attractor. We refer the reader to [Bessa et al., 2019] for more information.

\subsection{T-points}

The non-trivial dynamics we observe when $\lambda_{1}$ becomes positive emerge through a codimension three bifurcation of the Bykov attractor that exists when $\lambda_{1}=0=\lambda_{2}$. The first impact of a slight increase of the parameter $\lambda_{1}$ is the creation of Bykov cycles: every small enough $\lambda_{1}>0$ is a $T$-point since the map $f_{\left(\lambda_{1}, 0\right)}$ has a Bykov cycle. According to [Barrio et al., 2011; Bykov, 2000], for small $\lambda_{1}>0$ the line of points $\left(\lambda_{1}, 0\right)$ is surrounded by two spiral sheets arbitrarily close to the line within which we observe two families of homoclinic cycles of Shilnikov type, one attracting and another accumulated by horseshoes. These phenomena have also been explored in [Algaba et al., 2011] and [Lamb et al., 2005]; we will give more details about them in Section 10.

Among others, references [Aguiar et al., 2005; Algaba et al., 2011; Bykov, 2000; Fernández-Sánchez et al., 2002; Labouriau \& Rodrigues, 2012; Rodrigues \& Labouriau, 2014; Rodrigues, 2013] have been devoted to the study of $T$-points and the global bifurcations that they organize in the parameter space. For instance, as the two equilibria are saddle-foci, it is known that two spiral curves, corresponding to homoclinic connections of both equilibria, and a double infinity of spiral curves of saddle-node bifurcations of periodic orbits emerge from each $T$-point (see Figure 14).

Taking into account [Barrio et al., 2011, 2012; Gallas, 2010], this may justify the presence of shrimps and hubs in Figure 2. The mechanism of creation and destruction of dynamics when a third parameter is introduced, as done in [Algaba et al., 2010, 2011], is due to the lack of transversality of a curve of $T$-points in the three-dimensional parameter space.

Each $T$-point on the parameter plane is accumulated by infinitely many sequences of other $T$-points, from which an infinite number of bifurcation curves emanate [Labouriau \& Rodrigues, 2012]. This behavior is illustrated in Figure 4. The latter correspond to homoclinic loops of the saddle-foci, around which an infinite number of bifurcation curves spiral. Besides, subsidiary $T$-points emerge from the collision of the 


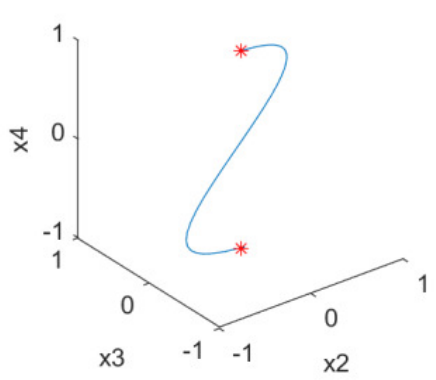

(a) 0

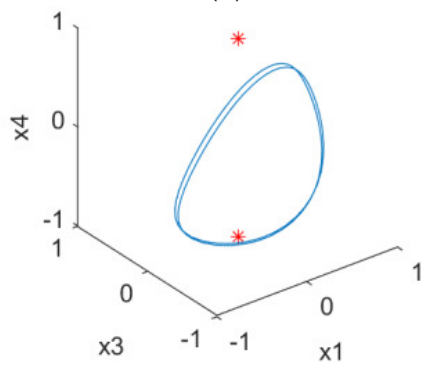

(d) 1.3

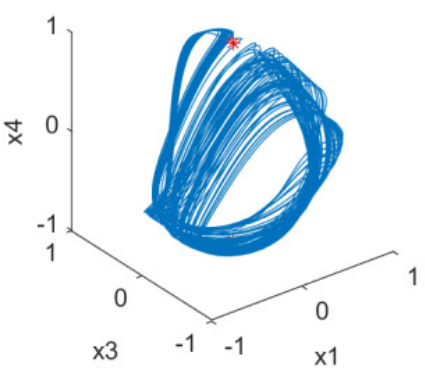

(g) 2.4

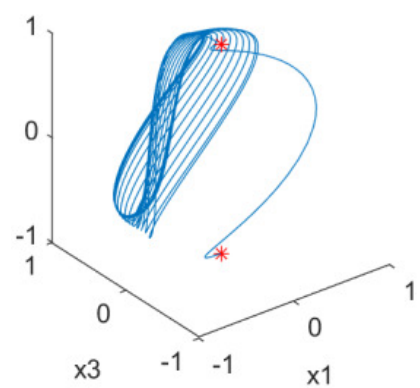

(b) 0.01

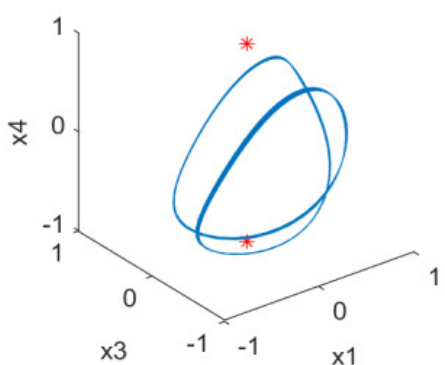

(e) 1.6

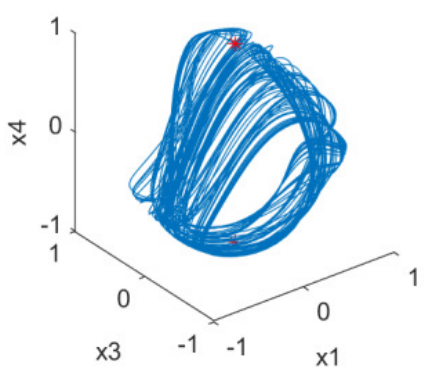

(h) 2.8

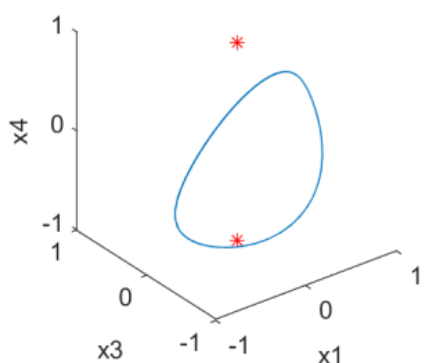

(c) 0.8

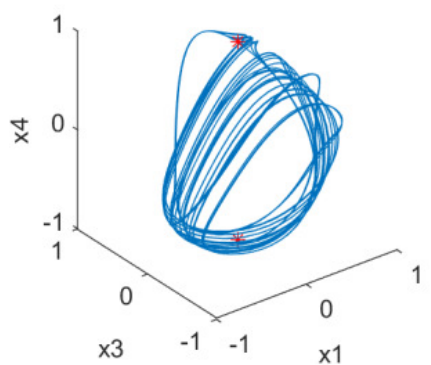

(f) 2

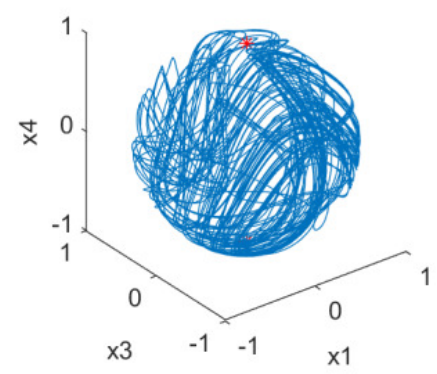

(i) 3.8

Fig. 12. Routes to chaos. $\lambda_{2}=0.5$ fixed. (a)-(k): different values of $\lambda_{1}$. Initial condition CI5 : $(0,0,1,0.1)$. Orbits for $t \in[0,10000]$. The stars represent the equilibria.

invariant two-dimensional manifolds of $\sigma_{1}$ and $\sigma_{2}$.

While the shape of some curves of global bifurcations can be predicted from the above mentioned papers when the parameters are close to a $T$-point, the results are no longer valid far from them. For instance, a parameter which is accumulated by a family of curves of $T$-points (cf. Figure 14(a)) seems to correspond to a $B$-point, as described in [Barrio et al., 2011], about which little information is available. Further numerical experiments ought to check if in the wedge of Figure 21 there is a $B$-point.

\subsection{Bifurcation routes}

When $\lambda_{1}>0$ and $\lambda_{2}$ becomes positive, all the symmetries are broken. In particular, the one-dimensional connection $\left[\sigma_{1} \rightarrow \sigma_{2}\right]$ is destroyed and we no longer expect to find infinitely many saturated horseshoes accumulating at $W^{u}\left(\sigma_{2}\right)$. Yet, due to the robustness of hyperbolicity, a finite number of those saturated horseshoes certainly survives under a small perturbation, whose extent is measured by $\lambda_{2}$. The same conclusion holds for the existing sinks, some of them still detected for small positive parameters $\lambda_{2}$. Most likely, this justifies part of both the grey and red regions we observe for $\lambda_{1}>0$ and very small $\lambda_{2}>0$ in the bifurcation diagram of Figure 16.

When $\lambda_{2}>0$ is increased, the size of the red region diminishes and we glimpse another dominion of 

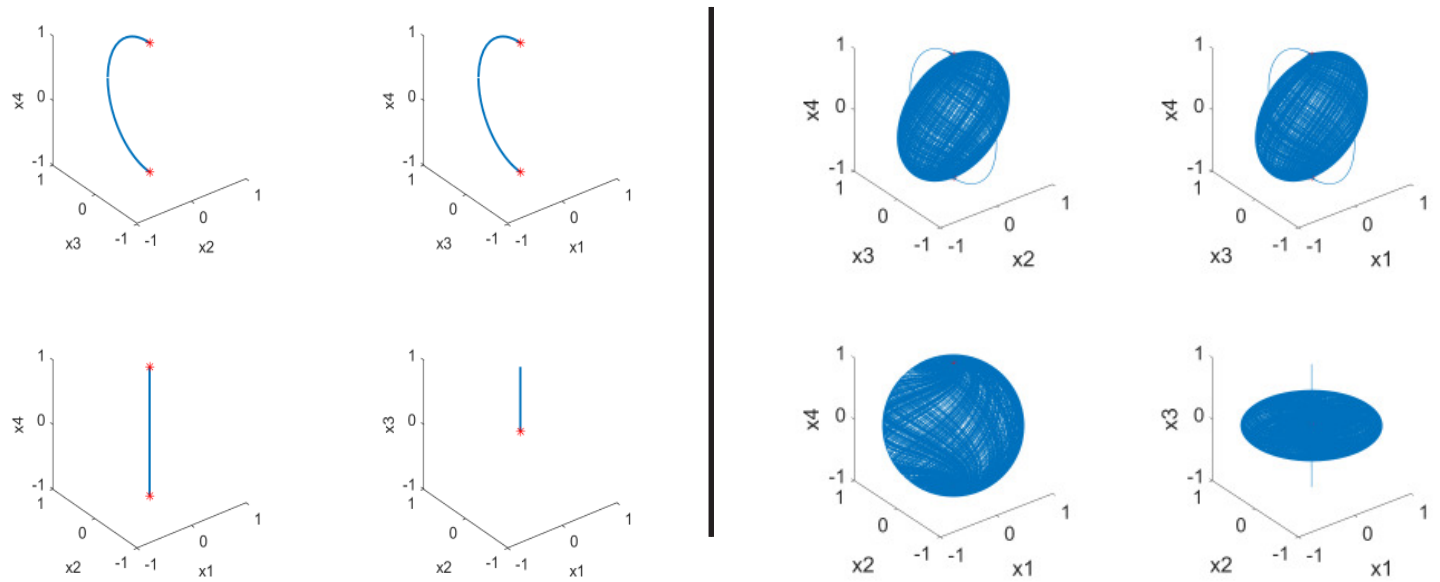

Fig. 13. Heteroclinic connections when $\lambda_{1}=0.01, \lambda_{2}=0, \alpha_{1}=1$ and $\alpha_{2}=-0.1$, realized by the orbit with initial condition CI1 (four images at left) and CI3 (right).

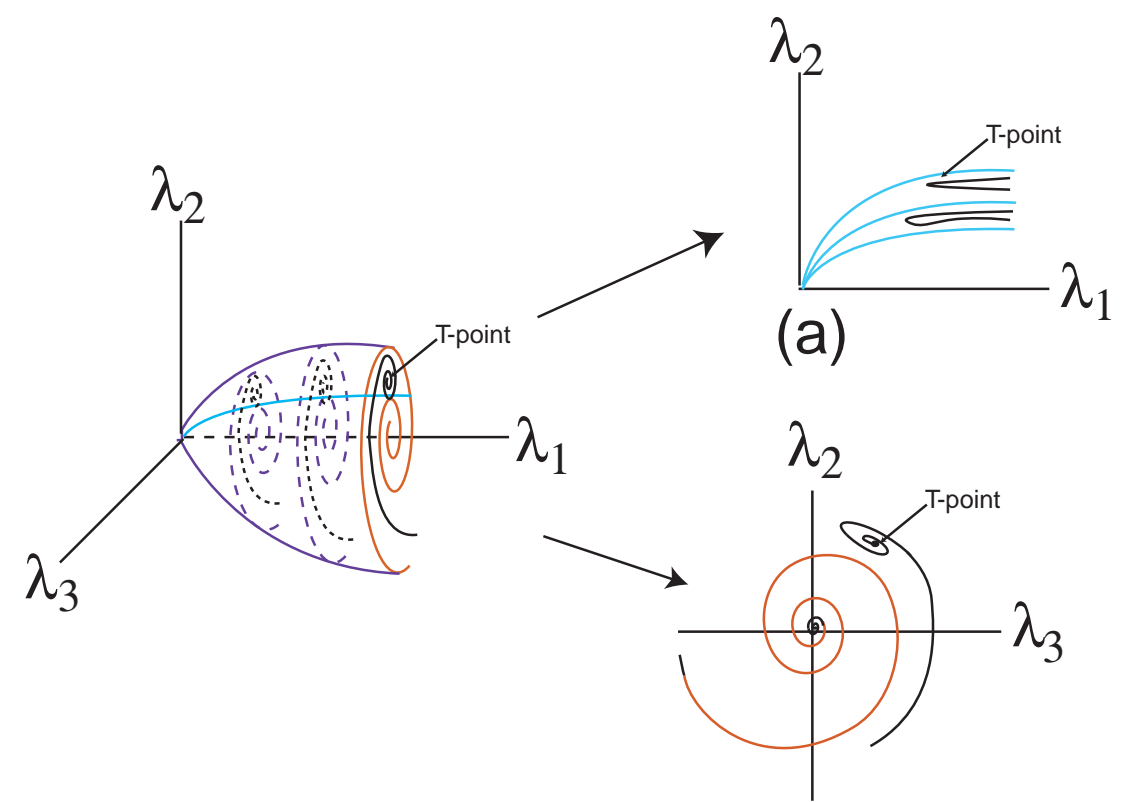

(b)

Fig. 14. Some bifurcation curves determined by $T$-points in the parameter space $\left(\lambda_{1}, \lambda_{2}, \lambda_{3}\right)$ and associated sections by: (a) the plane $\lambda_{3}=0 ;$ (b) a plane $\lambda_{1}=$ positive constant. It is worth while to compare this image with those of [Bykov, 2000] and [Algaba et al., 2010, 2011].

negative Lyapunov exponents. This may be due to the growth of the distance (see Figure 18) between the spirals mentioned in Lemma 1 , which is measured by $\lambda_{2}$, inducing the destruction of horseshoes, a bifurcation described in [Crovisier, 2002]. This phenomenon is accompanied by saddle-node and periodic doubling bifurcations and the emergence of attractors (cf. [Crovisier, 2002; Yorke \& Alligood, 1983]).

\section{9. $\lambda_{1}=0$ and $\lambda_{2}>0$}

In this section we will consider another possible bifurcation from $f_{(0,0)}$, namely the destruction of the $\Gamma_{1}$-symmetry. Thereby, the one-dimensional connection between the two equilibria $\sigma_{1}$ and $\sigma_{2}$ no longer exists. 

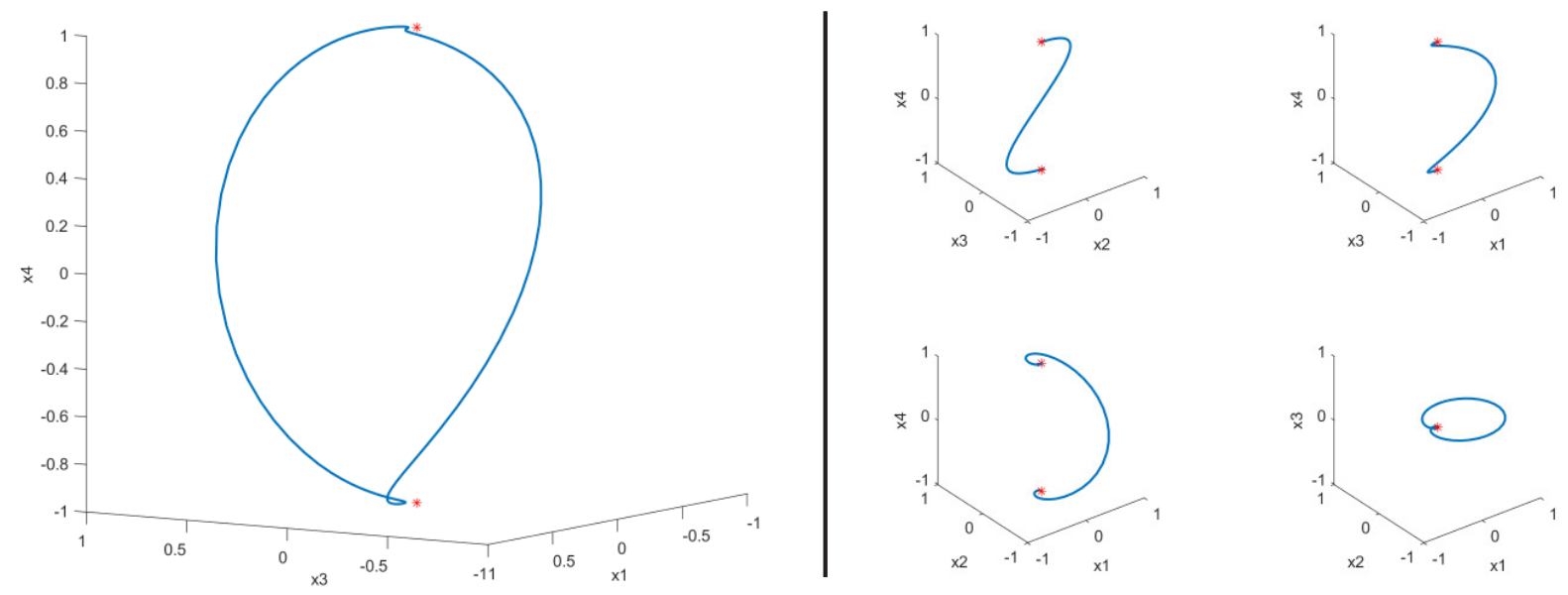

Fig. 15. Illustration of an attracting periodic orbit (left) and of a 1-dimensional heteroclinic connection when $\lambda_{1}=0$, $\lambda_{2}=0.01, \alpha_{1}=1$ and $\alpha_{2}=-0.1$ realized by the orbits of the initial conditions CI1 (left) and CI3 (right).

\subsection{Dynamics}

When $\lambda_{1}=0$ but $\lambda_{2}>0$, the two heteroclinic cycles of $\Sigma_{0}$, that existed in the symmetric configuration, disappear. Besides, the invariance of $\operatorname{Fix}\left(\Gamma_{2}\right)$ is not preserved and the geometry of the asymptotically stable heteroclinic network $\Sigma_{0}$ is altered. Nevertheless, near the basin of attraction of the original global attractor there still exists a global attracting structure: each cycle is replaced by an asymptotically stable periodic trajectory (a sink) which lies near the original attracting heteroclinic cycle (cf. [Labouriau \& Rodrigues, 2012]) and mimics the cycle. This type of bifurcation usually creates long-period periodic orbits that lie close to the heteroclinic cycles that existed before the perturbation, a property also studied in [Proctor \& Jones, 1988]. The corresponding period $\mathbb{P}$ and $\lambda_{2}$ are related by the formula

$$
\mathbb{P} \approx C \log \left(\frac{1}{\lambda_{2}}\right)
$$

where $C>0$ is a constant that depends on the behavior of the vector field away from two small neighborhoods of the equilibria and is usually very difficult to compute. Notice that, when $\lambda_{2}$ approaches zero, the sinks spend increasing intervals of time near the equilibria. Figure 15 (left) shows one of those sinks.

\subsection{Bifurcation routes}

According to [Labouriau \& Rodrigues, 2012], for small enough $\lambda_{2}$ the non-wandering set of the vector field $f_{\left(0, \lambda_{2}\right)}$ reduces to

$$
\Omega_{\left(0, \lambda_{2}\right)}=\left\{\sigma_{1}, \sigma_{2}\right\} \cup\{\text { two hyperbolic sinks }\}
$$

and the one-dimensional connection is broken. As $\Omega_{\left(0, \lambda_{2}\right)}$ is a hyperbolic set (though $f_{\left(0, \lambda_{2}\right)}$ is not a MorseSmale vector field due to the connection $\left[\sigma_{2} \rightarrow \sigma_{1}\right]$ ), we may find a small region $\mathcal{A}$ in the parameter space such that, if $\left(\lambda_{1}, \lambda_{2}\right) \in \mathcal{A}$, then the non-wandering set $\Omega_{\left(\lambda_{1}, \lambda_{2}\right)}$ of the vector field $f_{\left(\lambda_{1}, \lambda_{2}\right)}$ contains an analytic continuation of $\Omega_{\left(0, \lambda_{2}\right)}$. In particular, the two sinks mentioned in Subsection 9.1 survive for the parameters in $\mathcal{A}$, though their basins of attraction may shrink as $\lambda_{1}$ and $\lambda_{2}$ increase, as suggested by Figure 16.

We observe that, for the parameters inside the region $\mathcal{A}$ whose first coordinate $\lambda_{1}$ is positive, the stable manifold of $\sigma_{1}$ and the unstable manifold of $\sigma_{2}$ intersect transversely. Thus, they are ready to generate new non-wandering dynamics, and in fact Figure 16 seems to indicate that the boundary $\mathcal{C}$ of $\mathcal{A}$ separates a component with regular zero entropy dynamics from a chaotic one.

The diagram of Figure 16 suggests that the basin of the periodic attractor that captures the orbit of the chosen initial condition diminishes for smaller values of $\lambda_{2}$. One plausible explanation for this behavior may be the following: the emergence of positive Lyapunov exponents in this context is determined by 

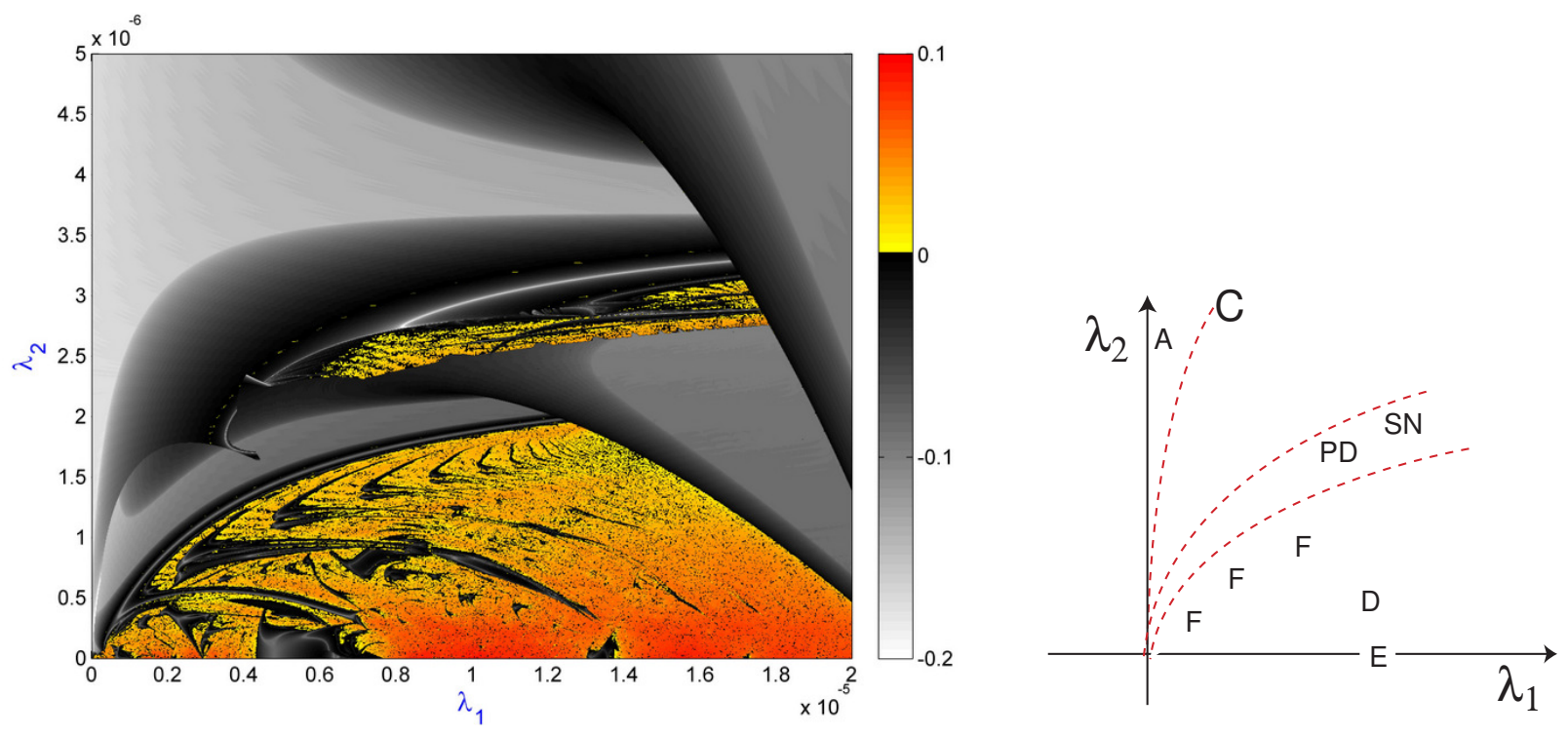

Fig. 16. Lyapunov exponents diagram in $\left(\lambda_{1}, \lambda_{2}\right) \in\left[0,2 \times 10^{-5}\right] \times\left[0,5 \times 10^{-6}\right]$ for the initial condition CI4: $=(-0.5,-0.129,-0.8807,-0.3013)$ and fixed parameters $\alpha_{1}=2.5$ and $\alpha_{2}=-0.1$. The picture is dominated by a grey/black region (most likely corresponding to sinks) and a red/orange domain, some of whose parameters exhibit saturated horseshoes coexisting with periodic attractors. The curve $C$ signalizes a bifurcation from regular to chaotic dynamics. Points colored in orange correspond to parameters for which the trajectory of CI4 exhibits a positive upper Lyapunov exponent; otherwise, the point is painted in grey or black.

the transverse intersection of the two-dimensional invariant manifolds of the equilibria, an event whose probability of occurrence is inversely proportional to the parameter $\lambda_{2}$ (see Figure 18). Besides, if we fix $\lambda_{2}>0$ and increase $\lambda_{1}$, we observe in Figure 16 that the periodic solution seems to lose attractiveness. An acceptable justification for this behavior may be the fact that this happens within a shrimp, inside which, according to [Barrio et al., 2012; Gallas, 2010], the sinks undergo period doubling and saddle-node bifurcations.

\section{0. $\lambda_{1}>0$ and $\lambda_{2}>0$}

In general, systems exhibiting heteroclinic or homoclinic connections are structurally unstable. Thereby, the knowledge of the bifurcation diagram in the region where $\lambda_{1}>0$ and $\lambda_{2}>0$ is so far quite fragmentary. A direct analysis of the return map is not feasible (at the moment) due to its extreme complexity. Using the numerical information, our goal is to propose some plausible explanations for the dynamical behavior of the vector field (1) when $\lambda_{1}>0, \lambda_{2}>0$ are sufficiently small. Not surprisingly, for small values of the parameters the solutions of the chosen initial conditions resemble the heteroclinic network $\Sigma_{0}$, as the time series of Figure 17 show.

For $\lambda_{1} \neq 0 \neq \lambda_{2}$, both $\mathbb{Z}_{2}\left(\Gamma_{1}\right)$ and $\mathbb{Z}_{2}\left(\Gamma_{2}\right)$ symmetries are broken. Besides the existence of uniformly hyperbolic horseshoes (see Section 8.1), there are homoclinic orbits of $\sigma_{1}$ and $\sigma_{2}$. As we will verify in Subsection 10.1, the presence of these homoclinic loops is a phenomenon which depends on the right combination of the parameters $\left(\lambda_{1}, \lambda_{2}\right)$. Using Lemma 1 we conclude that, when $\lambda_{1} \neq 0 \neq \lambda_{2}$, the tips of the double spirals $\Phi_{2}^{-1}\left(W^{s}\left(\sigma_{1}\right) \cap \operatorname{In}\left(\sigma_{2}\right)\right)$ and $\Psi_{1 \rightarrow 2} \circ \Phi_{1}\left(W^{u}\left(\sigma_{2}\right) \cap \operatorname{In}\left(\sigma_{1}\right)\right)$ are separated; and that, generically in the $\left(\lambda_{1}, \lambda_{2}\right)$-plane, the center of the first curve does not intersect the second spiral. Thus, the double spirals have only a finite number of intersections. Consequently, the number of heteroclinic connections from $\sigma_{1}$ to $\sigma_{2}$ is finite. Figure 18 shows a scheme of this process. 

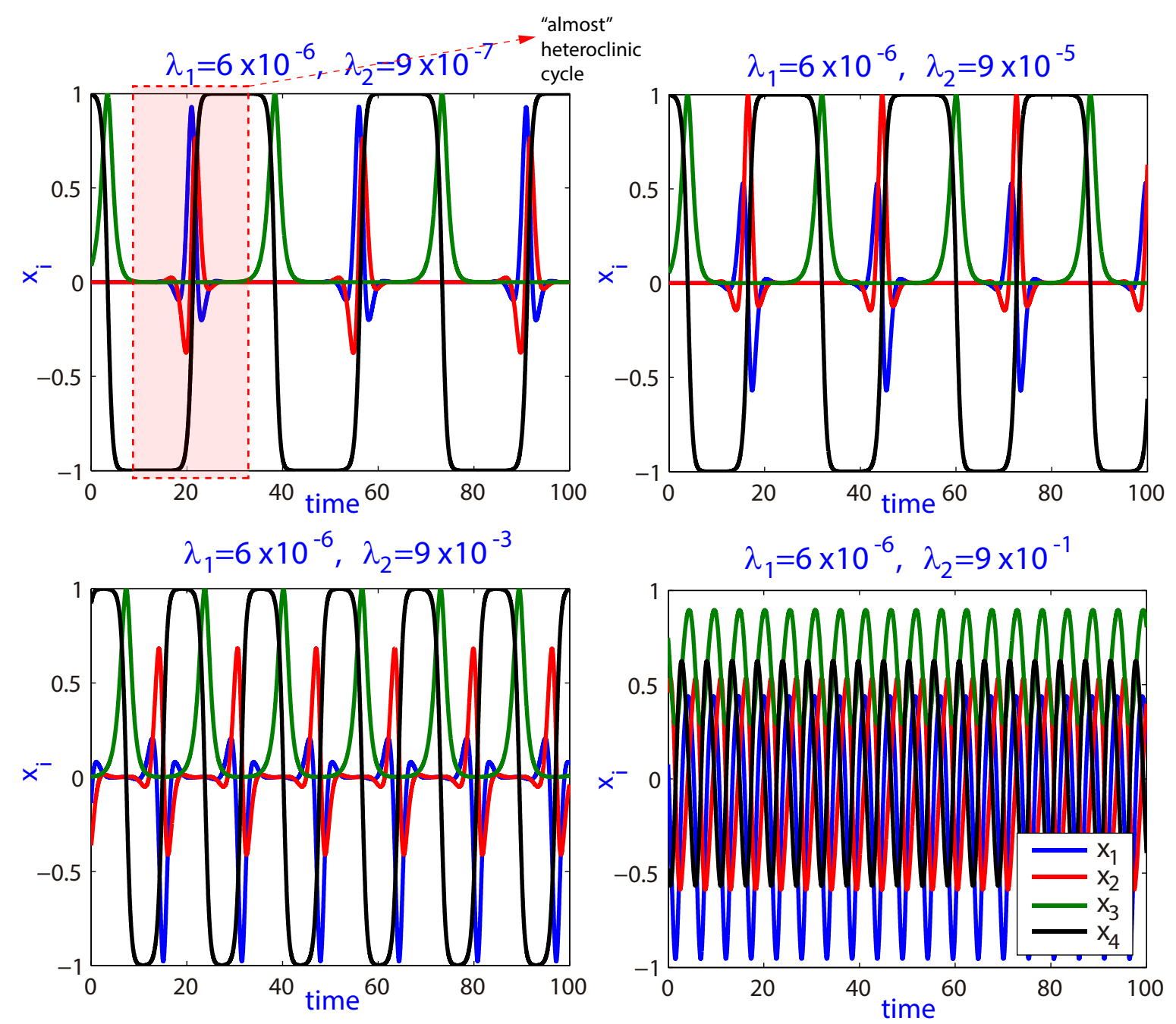

Fig. 17. Four $\omega$-limits (in this case, stable periodic orbits) for the parameter values $\alpha_{1}=1, \alpha_{2}=-0.1$ and $\lambda_{1}=6 \times 10^{-6}$ using the initial condition CI3: $=(0.01,0.01,0,-1)$. The parameter $\lambda_{2}$ changes from a value close to zero, $9 \times 10^{-7}$, to a large one, $9 \times 10^{-1}$, to illustrate the behaviour near the situation with heteroclinic connections and far from that regime. For $\lambda_{2}=9 \times 10^{-7}$ the trajectory seems to follow a periodic regime, visiting alternately neighborhoods of $\sigma_{1}$ and $\sigma_{2}$, spending there a large amount of time and making fast transitions outside them. This suggests the presence of a nearby heteroclinic cycle of the two saddle-foci. From the pictures, when $\lambda_{2}$ is slightly increased this heteroclinic cycle seems to be destroyed and it is likely, accordingly to the analysis in $\S 9.2$, that the simulated trajectory is now captured by a sink.

\subsection{Homoclinic connections}

We start observing that, for $\lambda_{1}>0$, there exists a homoclinic connection of $\sigma_{2}$ located in Out $\left(\sigma_{1}\right)$ if there are $\theta \in] \pi / 2,3 \pi / 2[$ and $k \in \mathbb{Z}$ such that

$$
\left\{\begin{array}{l}
\left(\lambda_{1} \sin (\theta)\right)^{\delta_{1}}=\lambda_{2} \\
-\frac{\omega_{1}}{E_{1}} \ln \left(\lambda_{1} \sin (\theta)\right)+\theta=2 k \pi .
\end{array}\right.
$$

When $\lambda_{2} \leq \lambda_{1}^{\delta_{1}}$, the previous equation is equivalent to

$$
\left\{\begin{array}{l}
\lambda_{1} \sin (\theta)=\lambda_{2}^{1 / \delta_{1}} \\
-\frac{\omega_{1}}{C_{1}} \ln \left(\lambda_{2}\right)+\arcsin \left(\frac{\lambda_{2}^{1 / \delta_{1}}}{\lambda_{1}}\right)=2 k \pi .
\end{array}\right.
$$




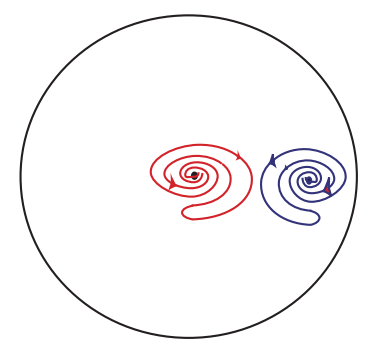

(a)

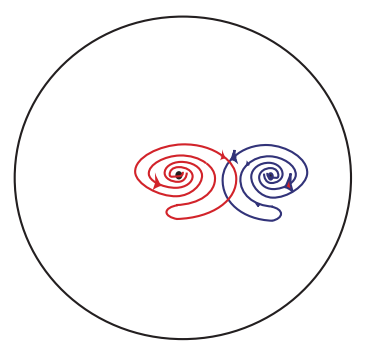

(b)

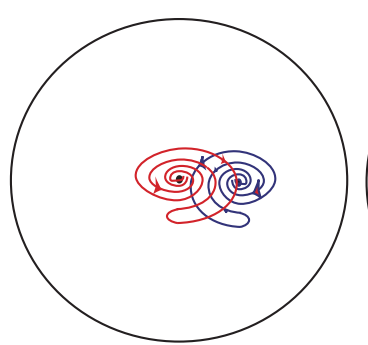

(c)

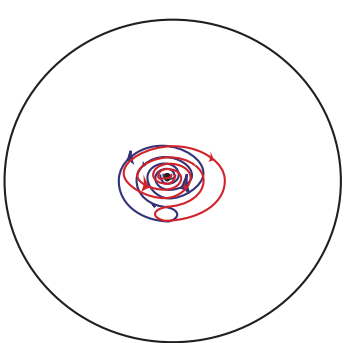

(d)

Fig. 18. The effect on $\operatorname{In}^{+}\left(\sigma_{2}\right)$ of decreasing $\lambda_{2}$, for a fixed $\lambda_{1}$. The two spiraling curves represent intersections of $W^{s}\left(\sigma_{1}\right)$ and $W^{u}\left(\sigma_{2}\right)$ with $\operatorname{In}^{+}\left(\sigma_{2}\right)$. (a) No new recurrent dynamics. (b)-(d) There exists a heteroclinic network where the number of connections increases to infinity as the distance between the tips of the spirals approaches zero.

For $\lambda_{1}>0$, if the homoclinic connection is performed by the tip point of the graph of $W^{u}\left(\sigma_{2}\right) \cap \operatorname{In}^{+}\left(\sigma_{1}\right)$, whose cartesian coordinates are $\left(\pi / 2, \lambda_{1}\right)$, then we have

$$
\left\{\begin{array}{l}
\lambda_{1}^{\delta_{1}}=\lambda_{2} \\
-\frac{\omega_{1}}{E_{1}} \ln \left(\lambda_{1}\right)=2 k \pi-\pi / 2
\end{array}\right.
$$

that is,

$$
\left\{\begin{array}{l}
\lambda_{1}^{\delta_{1}}=\lambda_{2} \\
\lambda_{1}=\exp \left[\frac{\pi / 2-2 k \pi}{\omega_{1} / E_{1}}\right]
\end{array}\right.
$$

Similarly, taking into account that

$$
\Phi_{2}^{-1}(1, \theta, z)=\left(z^{1 / \delta_{2}}, \theta+\frac{\omega_{2}}{C_{2}} \ln z, 1\right)
$$

one has a homoclinic connection of $\sigma_{1}$ in $\operatorname{In}\left(\sigma_{2}\right)$ if there are $\left.\theta \in\right] \pi / 2,3 \pi / 2[$ and $k \in \mathbb{Z}$ such that

$$
\left\{\begin{array}{l}
\left(\lambda_{1} \sin (\theta)\right)^{\delta_{2}^{-1}}=\lambda_{2} \\
\frac{\omega_{2}}{C_{2}} \ln \left(\lambda_{1} \sin (\theta)\right)+\theta=2 k \pi .
\end{array}\right.
$$

If the homoclinic connection is performed by the tip point of the graph of $W^{s}\left(\sigma_{1}\right) \cap \mathrm{Out}^{+}\left(\sigma_{2}\right)$, whose cartesian coordinates are $\left(\pi / 2, \lambda_{1}\right)$, then it satisfies the equations

$$
\left\{\begin{array}{l}
\lambda_{1}^{\delta_{2}^{-1}}=\lambda_{2} \\
\frac{\omega_{2}}{C_{2}} \ln \left(\lambda_{1}\right)=2 k \pi-\pi / 2
\end{array}\right.
$$

for some $k \in \mathbb{Z}$, that is,

$$
\left\{\begin{array}{l}
\lambda_{1}=\lambda_{2}^{\delta_{2}} \\
\lambda_{1}=\exp \left[\frac{2 k \pi-\pi / 2}{\omega_{2} / C_{2}}\right] .
\end{array}\right.
$$

In the plane $\left(\lambda_{1}, \lambda_{2}\right)$ some of the points which satisfy the previous equations are represented by the dotted line in Figure 19 (left).

In Figure 19, one sees a scheme of the red parabolic-type curves of Figure 20, whose interpretation we believe to be the following: for a fixed $\lambda_{1}>0$, there are infinitely many values of $\lambda_{2}>0$ for which we observe attracting homoclinic cycles of Shilnikov type. Although theoretically these phenomena occur along lines, in Figure 20 the red regions are thick. Most likely, this is due to the fact that, after the unfolding 


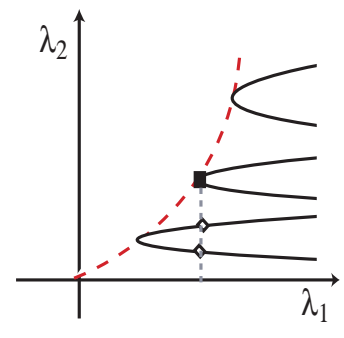

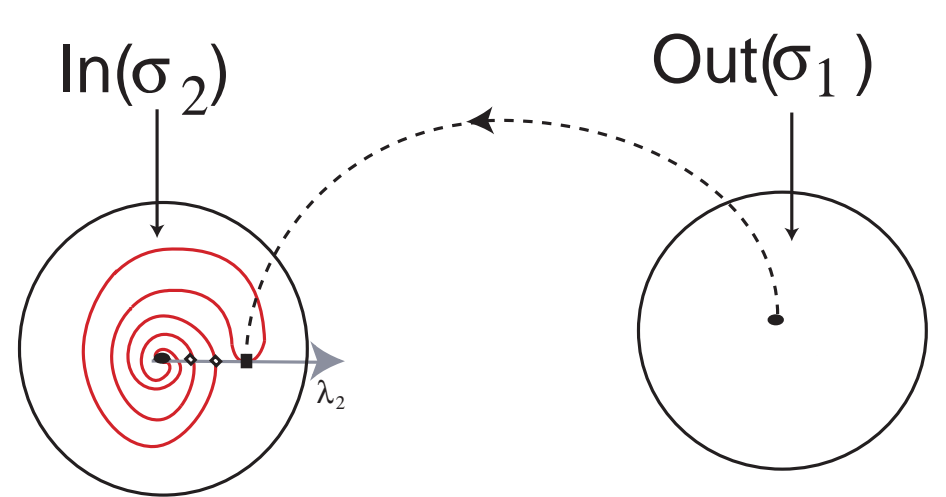

Fig. 19. Parameters with Shilnikov homoclinic cycles.

of an attracting Shilnikov homoclinic cycle, a sink with large period appears very close to the equilibrium; however, the numerical simulation is not able to distinguish them. The unfolding of such cycles has been studied by Shilnikov [Shilnikov, 1965, 1967] and Champneys et al. [Champneys et al., 2009].

It is known that the presence of Shilnikov homoclinic cycles of a saddle-focus is responsible for the global spiral organization of shrimps [Barrio et al., 2011; Bykov, 2000]. The appearance of a shrimp in a two-dimensional parameter space then leads to infinitely many self-similar shrimps and spirals, as well as super-stable periodic orbits and period doubling cascades towards the boundary crisis (see Figure 23).

We remark that a chaotic attractor with small rate of expansion may appear in a numerical simulation as an attracting periodic solution with very large period and a negative Lyapunov exponent very close to zero. In many cases, we do not have theoretical means to distinguish these quite different $\omega$-limits. Bearing in mind these limitations, one can detect in Figure 21 a classical period-doubling cascade (PD) giving rise to chaotic behavior. And in Figure 22, the computer drawing of two $\omega$-limits for the parameters $\lambda_{1}=3.706 \times 10^{-5}$ and $\lambda_{2}=8.211 \times 10^{-7}$ shows the coexistence of two chaotic attractors. This coexistence of attractors is quite common in several dynamical systems, as illustrated by the Rössler system in [Barrio et al., 2009] and it is originated from the collision of two or more parametric structures as shown in Figure 22 .

\section{Final Remarks}

Over recent years, a great deal of experimental works and modeling have been directed towards the identification of dynamical properties and invariants which may serve as key signatures uniting diverse nonlinear chaotic systems in a single class. One such class of low order dissipative systems has been recognized to possess one common, easily detected, pattern of spiral structures in a bi-parametric phase space. Despite the overwhelming number of studies reporting the occurrence of these spirals, much is still unknown about their underlying bifurcation scenarios, and a complete description of the dynamics is still out of reach.

In this work, we have reported analytical and numerical research regarding the relative abundance and structure of chaotic phases for the autonomous dissipative two-parameter family of vector fields described in (1), while it unfolds an attracting heteroclinic network between two saddle-foci. We investigated both local and global bifurcations in order to detect either hyperbolic or chaotic dynamics, and have clarified some relevant bifurcation phenomena. We have located a crisis region and proposed plausible mechanisms responsible for the transition to chaos through period doubling cascades, saddle-node bifurcations and Newhouse phenomena, among others. We conjecture that the spiraling patterns and the shrimp-like regions are universal features within systems unfolding homoclinic connections between saddle-foci.

Our focus has been on trajectories close to the collection of saturated horseshoes that are created when the original heteroclinic cycle is destroyed, in order to highlight the role of the invariant manifolds of the equilibria on the structure of the global attractor.

The following is a brief summary of what the numerical experiments evinced for positive parameter $\lambda_{1}$ and non-negative $\lambda_{2}$ : 


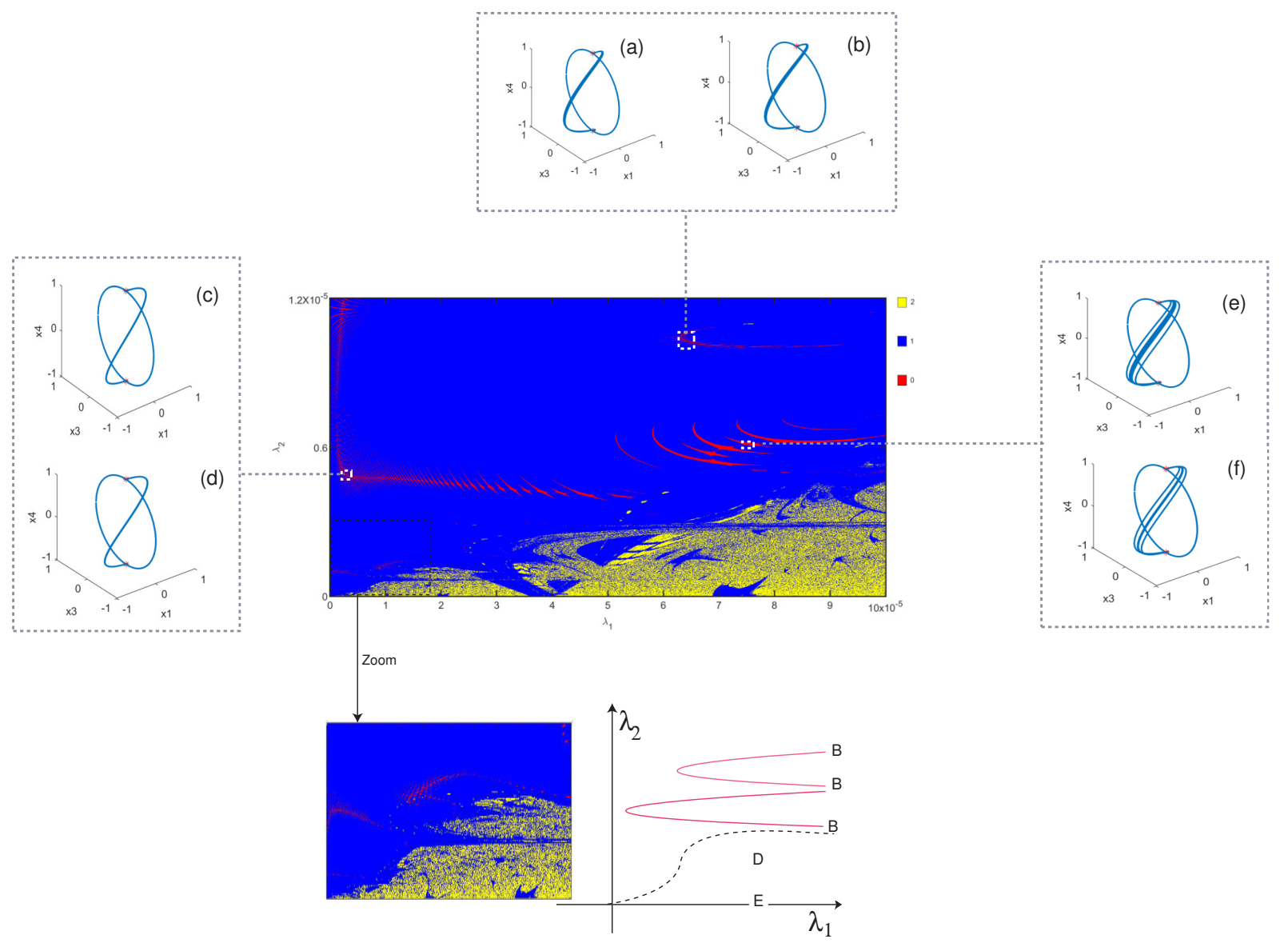

Fig. 20. Bifurcation diagram in the domain $\left(\lambda_{1}, \lambda_{2}\right) \in\left[0,10^{-4}\right] \times\left[0,1.2 \times 10^{-5}\right]$ for CI1:=(0,0,0.01, 0.9999). Each point is colored according to the number of positive Lyapunov exponents of the orbit with initial condition CI1: yellow (2), blue (1) and red (0), corresponding to an $\omega$-limit including a saddle-type orbit, a periodic solution or an equilibrium, respectively. The parabolic-type curves $B$ seems to correspond to parameters exhibiting attracting homoclinic cycles of one of the saddle-foci. Notice that the zoomed region is another representation of the third image of Figure 2. The values of $\lambda_{1}$ and $\lambda_{2}$ used to draw the attracting orbits are the following: (a) $\lambda_{1}=6.653 \times 10^{-5}, \lambda_{2}=1.007 \times 10^{-5}$; (b) $\lambda_{1}=6.933 \times 10^{-5}, \lambda_{2}=1.011 \times 10^{-5}$; (c) $\lambda_{1}=2.480 \times 10^{-5}$, $\lambda_{2}=5.560 \times 10^{-5}$; (d) $\lambda_{1}=3.110 \times 10^{-5}, \lambda_{2}=4.970 \times 10^{-5}$; (e) $\lambda_{1}=7.546 \times 10^{-5}, \lambda_{2}=5.850 \times 10^{-5}$; (f) $\lambda_{1}=8.962 \times 10^{-5}, \lambda_{2}=6.250 \times 10^{-5}$.

(1) The biparametric bifurcation diagrams depicted in Figures 2 and 20 clearly shows two regions corresponding to two dynamical phenomena that were theoretically predicted:

(a) a grey wedge whose left boundary is the $\lambda_{2}$-axis exhibiting the existence of an attracting periodic solution emerging from the destruction of a one-dimensional heteroclinic connection, between the two equilibria, which existed in an asymptotically stable network;

(b) a yellow/orange/red colored domain whose bottom boundary is the $\lambda_{1}$-axis, where we notice a prevalence of chaotic regimes, part of which are due to the destruction of a two-dimensional heteroclinic connection, yielding the transverse intersection of the two-dimensional invariant manifolds of the equilibria, while the setting is still close to fully symmetric context.

(2) The attractor of $\dot{x}=f_{\left(\lambda_{1}, \lambda_{2}\right)}(x)$ may not be minimal or even chain-transitive, and several attracting sets may coexist (cf. Figures 13 and 22).

(3) Although the general shape of the possible attracting set is unknown, numerical experiments detected the following forms:

- A closed curve (cf. Figures 2 and 21). 

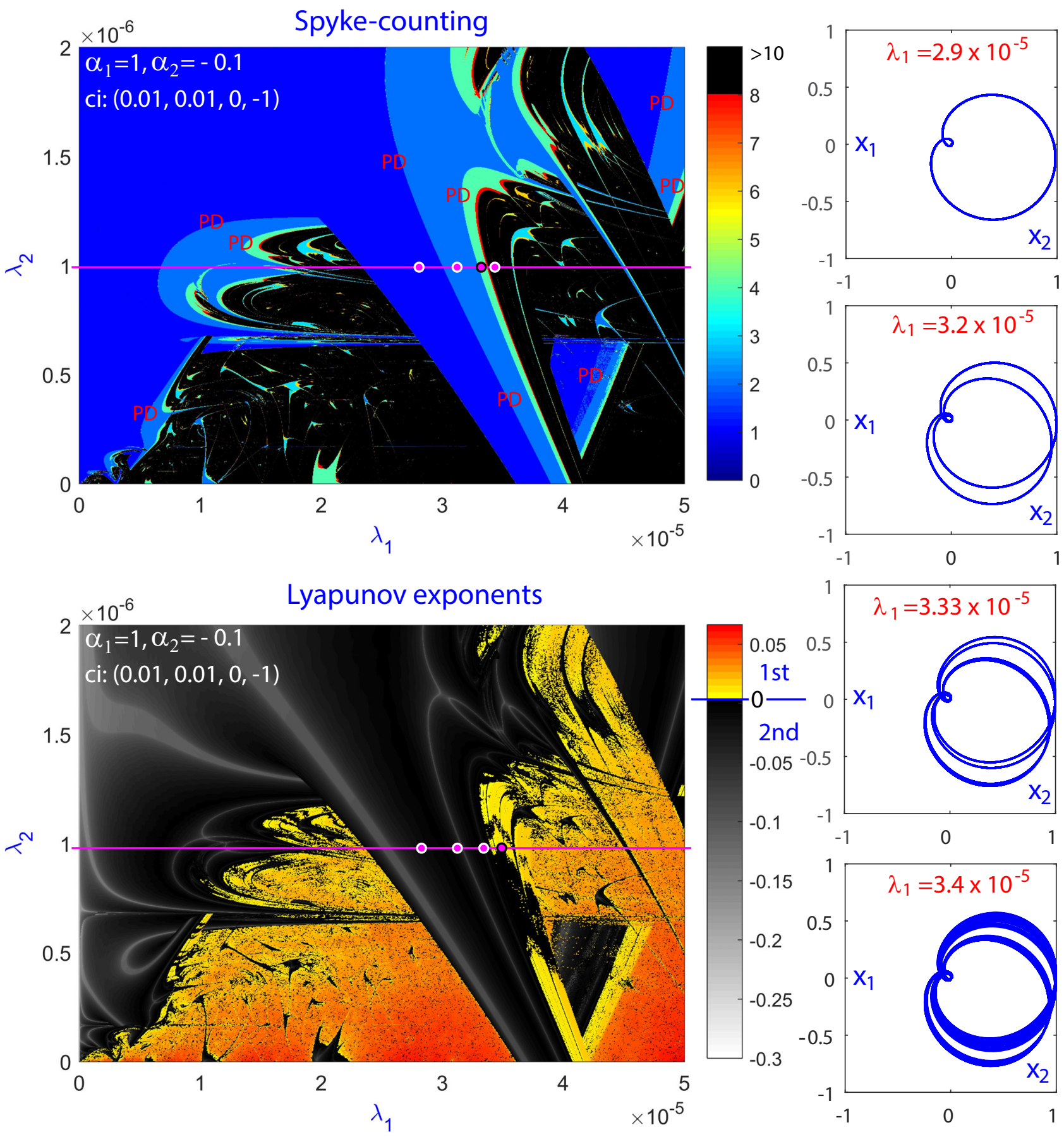

Fig. 21. Biparametric diagrams for $\left(\lambda_{1}, \lambda_{2}\right) \in\left[0,5 \times 10^{-4}\right] \times\left[0,2 \times 10^{-6}\right]$ and fixed parameters $\alpha_{1}=1$ and $\alpha_{2}=-0.1$ using the initial condition CI3: $=(0.01,0.01,0,-1)$. Upper image: each point is colored according to the number of loops (or spikes) around $\Sigma_{0}$, the Spyke-counting technique. Lower image: each point is colored according to the sign of the two biggest Lyapunov exponents along the orbit with initial conditions CI3. The column at right shows the projection on the plane $\left(x_{1}, x_{2}\right)$ of the trajectory starting at CI3 while the parameter $\lambda_{1}$ is increased moving along the pink horizontal line, showing a typical period-doubling cascade scenario (the last orbit exhibits almost chaotic behaviour).

- The union of a subset of the sphere $W^{u}\left(\sigma_{2}\right)$ and the connection $\left[\sigma_{1} \rightarrow \sigma_{2}\right]$ (cf. Figures 2, 13, 15 and $22)$.

(4) For $\lambda_{1}>0$, the detected attracting sets seem to be in $\overline{W^{u}\left(\sigma_{2}\right)}$, to vary continuously with the parameters and to collapse into $\Sigma_{0}$ as $\left(\lambda_{1}, \lambda_{2}\right)$ approaches $(0,0)$. 


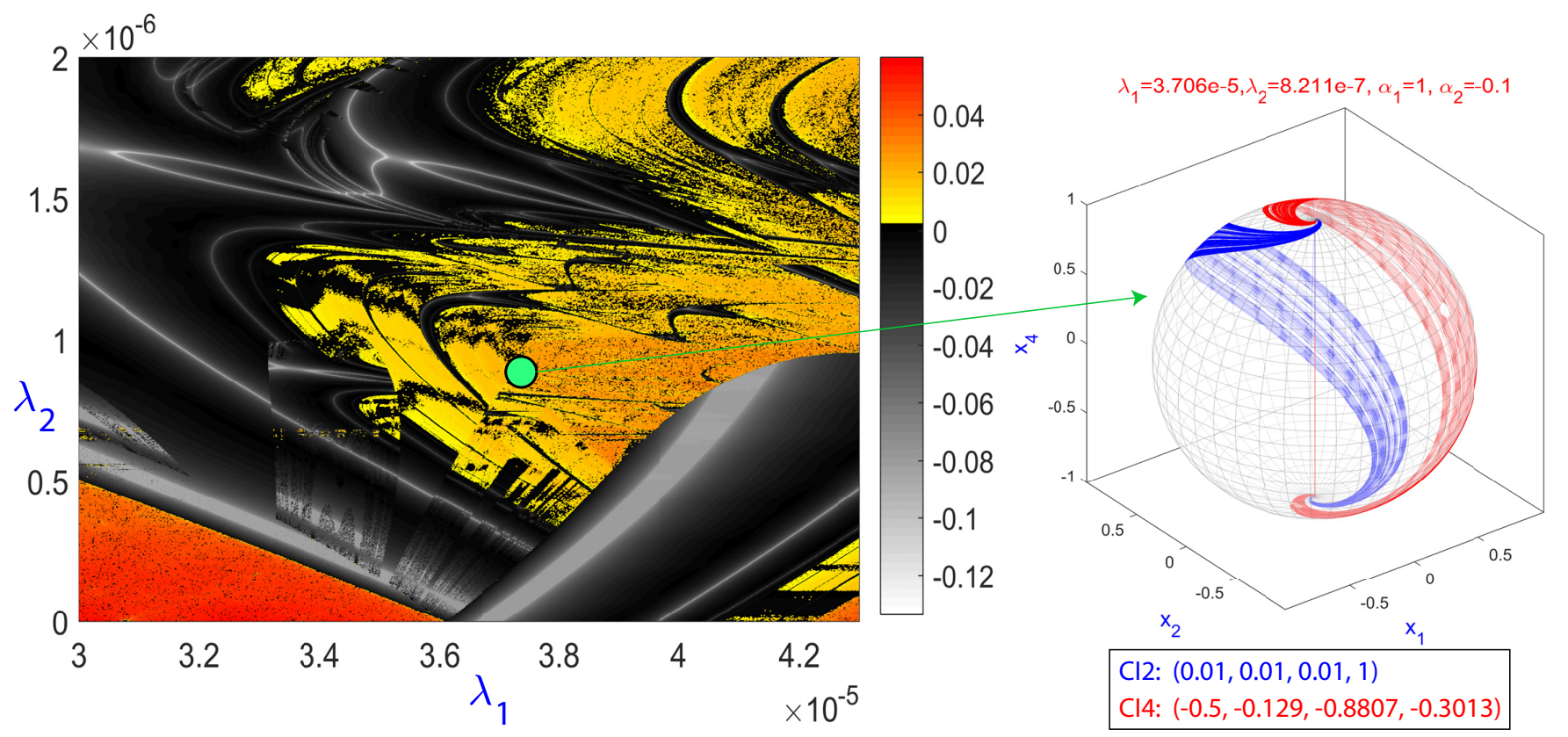

Fig. 22. On the left, we present a Lyapunov exponents biparametric diagram in the domain $\left(\lambda_{1}, \lambda_{2}\right) \in\left[3 \times 10^{-5}, 4.3 \times\right.$ $\left.10^{-5}\right] \times\left[0,2 \times 10^{-6}\right]$ for initial conditions CI2: $=(0.01,0.01,0.01,1)$ and fixed parameters $\alpha_{1}=1$ and $\alpha_{2}=-0.1$. On the right plot we show the approximations of the $\omega$-limit of the initial conditions CI2 and CI4 $((-0.5,-0.129,-0.8807,-0.3013))$ for the parameters $\lambda_{1}=3.706 \times 10^{5}$ and $\lambda_{2}=8.211 \times 10^{-7}$ (green dot in the diagram), showing the coexistence of at least two chaotic attractors for these values of the parameters.

\section{Acknowledgments}

RB was partially supported by the Spanish Research projects MTM2015-64095-P, PGC2018-096026-BI00, and by European Regional Development Fund and Diputación General de Aragón (E24-17R). MC and AR were partially supported by CMUP (UID/MAT/00144/2019), which is funded by FCT with national (MCTES) and European structural funds through the programs FEDER, under the partnership agreement PT2020. AR also acknowledges financial support from Program INVESTIGADOR FCT (IF/00107/2015). Part of this work has been written during AR's stay in Nizhny Novgorod University, supported by the grant RNF 14-41-00044. Luísa Castro has been financed by National Funds through the Portuguese funding agency FCT - Fundação para a Ciência e a Tecnologia within the project UID/EEA/50014/2019.

\section{References}

Abad, A., Barrio, A. R., Blesa, F. \& Rodríguez, M. [2012] Algorithm 924: TIDES, a Taylor Series Integrator for Differential Equations, ACM Trans. Math. Softw 39:1, 1-28.

Aguiar, M. A. D., Castro, S. B. S. D. \& Labouriau, I. S. [2005] Dynamics near a heteroclinic network, Nonlinearity 18, 391-414.

Aguiar, M. A. D., Castro, S. B. S. D. \& Labouriau, I. S. [2006] Simple vector fields with complex behavior, Internat. J. Bifur. Chaos Appl. Sci. Engrg. 16:2, 369-381.

Aguiar, M. A. D., Labouriau, I. S. \& Rodrigues, A. A. P. [2010] Switching near a heteroclinic network of rotating nodes, Dyn. Sys. Int. J. 25:1, 75-95.

Algaba, A., Fernández-Sánchez, F., Merino, M. \& Rodríguez-Luis, A. [2010] Analysis of the T-point Hopf bifurcation with $Z_{2}$-symmetry. Application to Chua's equation, Internat. J. Bifur. Chaos Appl. Sci. Engrg. 20:4 (2010) 979-993.

Algaba, A., Fernández-Sánchez, F., Merino, M. \& Rodríguez-Luis, A. [2011] Structure of saddle-node and cusp bifurcations of periodic orbits near a non-transversal T-point, Nonlinear Dynam. 63:3, 455-476.

Barrio, R., Blesa, F. \& Serrano, S. [2009] Qualitative analysis of the Rössler equations: Bifurcations of limit cycles and chaotic attractors, Physica D: Nonlinear Phenomena 238:13, 1087-1100. 

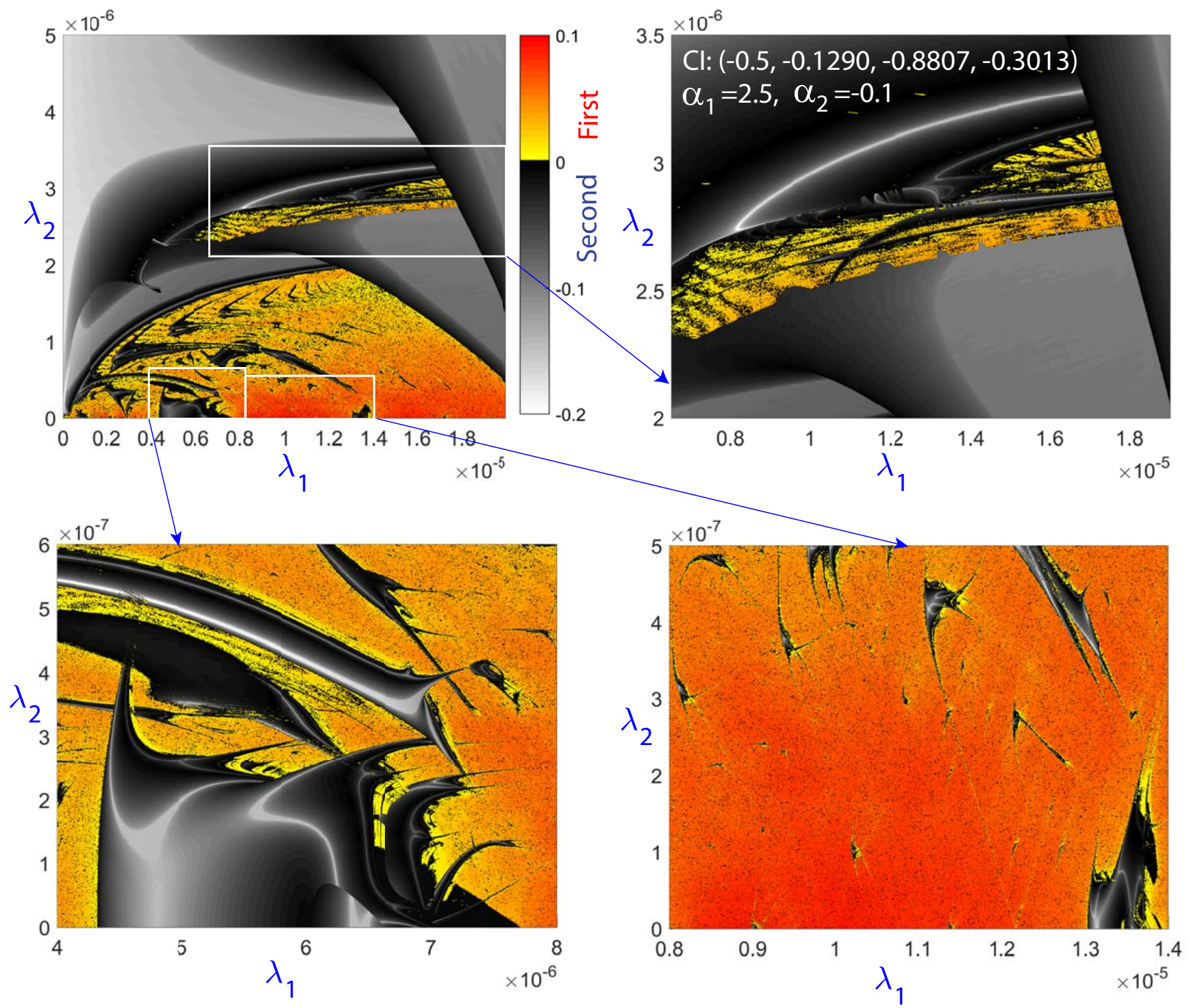

Fig. 23. Shrimps. Top left: Lyapunov exponents biparametric diagram in the domain $\left(\lambda_{1}, \lambda_{2}\right) \in\left[0,2 \times 10^{-5}\right] \times\left[0,5 \times 10^{-6}\right]$ using the initial condition CI4: $=(-0.5,-0.129,-0.8807,-0.3013)$ and fixed parameters $\alpha_{1}=2.5$ and $\alpha_{2}=-0.1$. The other plots show different magnifications of shrimp structures.

Barrio, R., Blesa, F., Serrano, S. \& Shilnikov, A. [2011] Global organization of spiral structures in biparameter space of dissipative systems with Shilnikov saddle-foci, Physical Review E 84, 035201.

Barrio, R., Blesa, F. \& Serrano, S. [2012] Topological changes in periodicity hubs of dissipative systems, Physical Review Letters 108:21, 214102.

Bessa, M., Carvalho, M. \& Rodrigues, A. A. P. [2019] The role of the saddle-foci on the structure of a Bykov attracting set, Qual. Theory Dyn. Syst. (accepted).

Bykov, V. V. [1980] On the structure of bifurcations sets of dynamical systems that are systems with a separatrix contour containing saddle-focus, Methods of Qualitative Theory of Differential Equations, Gorky University (in Russian), 44-72.

Bykov, V. V. [2000] Orbit structure in a neighbourhood of a separatrix cycle containing two saddle-foci, Amer. Math. Soc. Transl. 200, 87-97.

Carvalho, M. \& Rodrigues, A. A. P. [2018] Complete set of invariants for a Bykov attractor, Regular \& Chaotic Dynamics 23:3, 227-247.

Champneys, A. R., Kirk, V., Knobloch, E., Oldeman, B. E. \& Rademacher, J. D. M. [2009] Unfolding a 
tangent equilibrium-to-periodic heteroclinic cycle, SIAM J. Applied Dyn. Systems 8:3, 1261-1304.

Crovisier, S. [2002] Saddle-node bifurcations for hyperbolic sets, Ergod. Th. \& Dynam. Sys. 22, 1079-1115.

Deng, B. [1993] Homoclinic twisting bifurcations and cusp horseshoe maps, J. Dyn. Diff. Equations 5:3, 417-467.

Fernández-Sánchez, F., Freire, E. \& Rodríguez-Luis, A. [2002] T-points in a $Z_{2}$-symmetric electronic oscillator. I. Analysis, Nonlinear Dynam. 28:1, 53-69.

Gallas, J. [2010] The structure of infinite periodic and chaotic hub cascades in phase diagrams of simple autonomous flows, Internat. J. Bifur. Chaos Appl. Sci. Engrg. 20:2, 197-211.

Gonchenko, S. V., Shilnikov, L. P. \& Turaev, D. V. [1996] Dynamical phenomena in systems with structurally unstable Poincaré homoclinic orbit, Chaos 6:1, 15-31.

Guckenheimer, J. \& Worfolk, P. [1992] Instant chaos, Nonlinearity 5:6, 1211-1222.

Guckenheimer, J., Meloon, B. A., Myers, M. R., Wicklin, F.J. \& Worfolk, P. [1997] DsTool: A Dynamical System Toolkit with an Interactive Graphical Interface - User's Manual, Version Tk Draft. (Center for Applied Mathematics, Cornell University).

Homburg, A. J. \& Sandstede, B. [2010] Homoclinic and Heteroclinic Bifurcations in Vector Fields, Handbook of Dynamical Systems. (North Holland, Amsterdam), Vol. 3, pp. 379-524.

Krupa, M. \& Melbourne, I. [1995] Asymptotic stability of heteroclinic cycles in systems with symmetry II, Ergod. Th. E Dynam. Sys. 15, 121-147.

Labouriau, I. S. \& Rodrigues, A. A. P. [2012] Global generic dynamics close to symmetry, J. Diff. Eqs. 253:8, 2527-2557.

Labouriau, I. S. \& Rodrigues, A. A. P. [2016] Global bifurcations close to symmetry, J. Math. Anal. Appl. 444:1, 648-671.

Lamb, J. S. W., Teixeira, M. A. \& Webster, K. N. [2005] Heteroclinic bifurcations near Hopf-zero bifurcation in reversible vector fields in $\mathbb{R}^{3}$, J. Diff. Eqs. 219, 78-115.

Palis, J. \& Takens, F. [1995] Hyperbolicity and Sensitive Chaotic Dynamics at Homoclinic Bifurcations: Fractal Dimensions and Infinitely Many Attractors in Dynamics. (Cambridge University Press).

Proctor, M. \& Jones, C. [1988] The interaction of two spatially resonant patterns in thermal convection, J. Fluid Mech. 188, 301-335.

Rodrigues, A. A. P. [2013] Repelling dynamics near a Bykov cycle, J. Dyn. Diff. Equations 25:3, 605-625.

Rodrigues, A. A. P. \& Labouriau, I. S. [2014] Spiralling dynamics near heteroclinic networks, Physica D 268, 34-49.

Shilnikov, L. P. [1965] A case of the existence of a denumerable set of periodic motions, Sov. Math. Dokl 6, $163-166$.

Shilnikov, L. P. [1967] The existence of a denumerable set of periodic motions in four dimensional space in an extended neighbourhood of a saddle-focus, Sov. Math. Dokl. 8:1, 54-58.

Swift, J. W. [1988] Hopf bifurcation with the symmetry of the square, Nonlinearity 1, 333-377.

Wiggins, S. [1988] Global Bifurcations and Chaos. Analytical Methods, Applied Mathematical Sciences. (Springer-Verlag, New York), 73.

Wolf, A., Swift, J. B., Swinney, H. L. \& Vastano, J. A. [1985] Determining Lyapunov exponents from a time series, Physica D 16, 285-317.

Yorke, J.A. \& Alligood, K. T. [1983] Cascades of period-doubling bifurcations: A prerequisite for horseshoes, Bull. Amer. Math. Soc. (N.S.) 9:3, 319-322. 